\title{
Article
}

\section{Lagrangian Curve Flows on Symplectic Spaces}

\author{
Chuu-Lian Terng ${ }^{1, *}$ and Zhiwei $\mathrm{Wu}^{2}$ (D) \\ 1 Department of Mathematics, University of California at Irvine, Irvine, CA 92697, USA \\ 2 School of Mathematics (Zhuhai), Sun Yat-Sen University, Zhuhai 519082, China; wuzhiwei3@mail.sysu.edu.cn \\ * Correspondence: cterng@math.uci.edu
}

check for updates

Citation: Terng, C.L.; Wu, Z.

Lagrangian Curve Flows on Symplectic Spaces. Symmetry 2021, 13, 298. https://doi.org/10.3390/ sym 13020298

Academic Editor: Valentin Lychagin Received: 10 January 2021 Accepted: 3 February 2021 Published: 9 February 2021

Publisher's Note: MDPI stays neutral with regard to jurisdictional clai$\mathrm{ms}$ in published maps and institutional affiliations.

Copyright: () 2021 by the authors. Licensee MDPI, Basel, Switzerland. This article is an open access article distributed under the terms and conditions of the Creative Commons Attribution (CC BY) license (https:// creativecommons.org/licenses/by/ $4.0 /)$.

\begin{abstract}
A smooth map $\gamma$ in the symplectic space $\mathbb{R}^{2 n}$ is Lagrangian if $\gamma, \gamma_{x}, \ldots, \gamma_{x}^{(2 n-1)}$ are linearly independent and the span of $\gamma, \gamma_{x}, \ldots, \gamma_{x}^{(n-1)}$ is a Lagrangian subspace of $\mathbb{R}^{2 n}$. In this paper, we (i) construct a complete set of differential invariants for Lagrangian curves in $\mathbb{R}^{2 n}$ with respect to the symplectic group $S p(2 n)$, (ii) construct two hierarchies of commuting Hamiltonian Lagrangian curve flows of C-type and A-type, (iii) show that the differential invariants of solutions of Lagrangian curve flows of C-type and A-type are solutions of the Drinfeld-Sokolov's $\hat{C}_{n}^{(1)}-\mathrm{KdV}$ flows and $\hat{A}_{2 n-1}^{(2)}-\mathrm{KdV}$ flows respectively, (iv) construct Darboux transforms, Permutability formulas, and scaling transforms, and give an algorithm to construct explicit soliton solutions, (v) give bi-Hamiltonian structures and commuting conservation laws for these curve flows.
\end{abstract}

Keywords: Lagrangian curve flows; KdV type hierarchies; Darboux transforms

\section{Introduction}

The modern theory of soliton equations dates from the famous numerical computation of the interaction of solitary waves of the Korteweg-de Vries (KdV) equation by Zabusky and Kruskal [1] in 1965. In 1967, Gardner, Green, Kruskal, and Miura [2] applied the Gelfand-Levitan's inverse scattering transform of the one-dimensional linear Schrödinger operator to solve the Cauchy problem for rapidly decaying initial data for the KdV equation. In 1968, Lax [3] introduced the Lax-pair for KdV. Zakharov and Faddeev [4] gave a Hamiltonian formulation of $\mathrm{KdV}$, and proved that $\mathrm{KdV}$ is completely integrable by finding action-angle variables. Zakharov and Shabat [5] found a Lax pair of $2 \times 2$ first order differential operators for the non-linear Schrödinger equation (NLS), Adler-Kostant-Symes gave a method to construct completely integrable Hamiltonian systems using splitting of Lie algebras (cf. [6-9]), Kupershmidt-Wilson [10] constructed $n \times n$ modified KdV (mKdV) using a loop algebra, and finally Drinfeld-Sokolov [11] gave a general method to construct soliton hierarchies from affine Kac-Moody algebras. In particular, soliton equations have many remarkable properties including: a Lax pair, infinite families of explicit soliton solutions, Bäcklund and Darboux transformations that generate new solutions from a given one by solving a first order system, a permutability formula to superpose solutions, a rational loop group action, a scattering theory and an inverse scattering transform to solve the Cauchy problem, a bi-Hamiltonian structure, and infinitely many commuting Hamiltonians. For more detail and references, we refer readers to the following books and survey articles: [11-18].

Soliton equations are also found in classical differential geometry: the sine-Gordon equation (SGE) arose first through the theory of surfaces of negative constant Gauss curvature in $\mathbb{R}^{3}$, and the reduced 3-wave equation can be found in Darboux's work [19] on triply orthogonal coordinate systems of $\mathbb{R}^{3}$. These equations were rediscovered later independently of their geometric history. The main contribution of the classical geometers lies in their methods for constructing explicit solutions of these equations from geometric transformations.

There are many classes of submanifolds in space forms and symmetric spaces whose Gauss-Codazzi equations are soliton equations. For example, the Gauss-Codazzi equations 
for the following classes of submanifolds are soliton equations: n-dimensional submanifolds of constant sectional curvature -1 in in $\mathbb{R}^{2 n-1}$ (cf. [20,21]), isometric immersions of space forms in space forms (cf. [22,23]), flat Lagrangian submanifolds in $\mathbb{R}^{2 n}$ [24], conformally flat submanifolds in spheres [25], and isothermic submanifolds in $\mathbb{R}^{n}$ (cf. [26-28]). For a survey of submanifold geometry and related soliton equations see [29].

Next we discuss how curve flows appeared in soliton theory. In 1906, da Rios, a student of Levi-Civita, wrote a master's thesis, in which he modeled the movement of a thin vortex by the motion of a curve propagating in $\mathbb{R}^{3}$ along its binormal with curvature as speed, i.e.,

$$
\gamma_{t}=k \mathbf{b} \text {. }
$$

This is the vortex filament equation (VFE). It was much later, in 1971, that Hasimoto showed in [30] the equivalence of VFE with the NLS,

$$
q_{t}=i\left(q_{x x}+2|q|^{2} q\right) .
$$

In fact, if $\gamma(x, t)$ is a solution of VFE, then there exists a function $\theta(t)$ such that

$$
q(x, t)=k(x, t) \exp \left(i\left(\theta(t)-\int_{-\infty}^{x} \tau(s, t) \mathrm{d} s\right)\right)
$$

is a solution of the NLS, where $k, \tau$ are the curvature and torsion of the curve. This correspondence between the VFE and NLS given above uses the Frenet frame. If we use the parallel normal frame, then the correspondence can be stated as follows: If $\gamma$ is a solution of the VFE, then there exists an orthonormal moving frame $g=\left(e_{1}, e_{2}, e_{3}\right): \mathbb{R}^{2} \rightarrow S O(3)$ such that

$$
g^{-1} g_{x}=\left(\begin{array}{ccc}
0 & -k_{1} & -k_{2} \\
k_{1} & 0 & 0 \\
k_{2} & 0 & 0
\end{array}\right),
$$

and $q=k_{1}+i k_{2}$ is a solution of the NLS, where $e_{1}(\cdot, t)$ is tangent to the curve $\gamma(\cdot, t), e_{2}(\cdot, t)$ and $e_{3}(\cdot, t)$ are parallel normal fields along $\gamma(\cdot, t)$, and $k_{1}(\cdot, t)$ and $k_{2}(\cdot, t)$ are the principal curvatures along $e_{2}(\cdot, t)$ and $e_{3}(\cdot, t)$ respectively. Since the NLS is a soliton equation, we can use techniques in soliton theory to study geometric and Hamiltonian aspects of the VFE.

The NLS admits an so(3) valued Lax pair with phase space $C^{\infty}(\mathbb{R}, V)$, where

$$
V=\left\{\left(\begin{array}{ccc}
0 & -k_{1} & -k_{2} \\
k_{1} & 0 & 0 \\
k_{2} & 0 & 0
\end{array}\right) \mid k_{1}, k_{2} \in \mathbb{R}\right\} .
$$

Please note that the differential invariants constructed from the parallel frames for curves in $\mathbb{R}^{3}$ lie in $C^{\infty}(\mathbb{R}, V)$. Hence a good way to construct integrable curve flows on a homogeneous space $M=G \cdot p_{0}=G / H$ is to find a class of curves in $G / H$, which has a moving frame $g: \mathbb{R} \rightarrow G$ so that $\gamma=g \cdot p_{0}, g^{-1} g_{x}$ gives a complete set of differential invariants, and $g^{-1} g_{x}$ lies in the phase space of a soliton equation. A more detailed discussion of how to use this scheme to construct integrable curve flows can be found in [31].

There are many recent works on integrable geometric curve flows in homogeneous spaces. For example, Langer-Perline studied Poisson structures and local geometric invariants of the VFE in $[32,33]$, and constructed curve flows that relate to Fordy-Kulish NLS type hierarchies associated with Hermitian symmetric spaces in [34]. Doliwa-Santini constructed curve flows in $\mathbb{R}^{2}$ and $\mathbb{R}^{3}$ that give the $\mathrm{mKdV}$ and NLS respectively in [35]. Ferapontov gave hydro-dynamic type curve flows on homogeneous isoparametric hypersurfaces in sphere in [36]. Yasui-Sasaki studied the integrability of the VFE in [37]. Chou-Qu constructed integrable curve flows in affine plane in [38] and integrable curve flows in the plane for all Klein geometries in [39]. Anco constructed integrable curve flows on the symmetric space $\frac{U}{K}$ in [40]. Sanders-Wang studied curve flows in $\mathbb{R}^{n}$ whose curvatures 
are solutions of the vector $\mathrm{mKdV}$ in [41]. Terng-Thorbergsson constructed curve flows on Adjoint orbits of a compact Lie group $G$ that relate to the n-wave equation associated with $G$ in [42], Terng-Uhlenbeck explained the relation between the Schrödinger flow on compact Hermitian symmetric space and the Fordy-Kulish NLS system and wrote down a bi-Hamiltonian structure, geometric conservation laws, and commuting curve flows in [43] for the Schrödinger flows. Terng constructed Darboux transforms and explicit soliton solutions of the Airy curve flow in $\mathbb{R}^{n}$ in [44]. Mari Beffa gave natural Poisson structures on semi-simple homogeneous spaces and discussed their relations to integrable curve flows in $[45,46]$. Readers are referred to these papers for more references.

Drinfeld and Sokolov in [11] associated with each affine Kac-Moody algebra $\hat{\mathcal{G}}$ a hierarchy of soliton equations of KdV type, which will be called the $\hat{\mathcal{G}}$-KdV hierarchy. It was proved in [11] that the KdV hierarchy is the $\hat{A}_{1}^{(1)}-\mathrm{KdV}$ hierarchy and the Gelfand-Dickey hierarchy is the $\hat{A}_{n-1}^{(1)}$-KdV hierarchy.

There are recent works on integrable curve flows on flat spaces whose differential invariants satisfy the $\hat{\mathcal{G}}-\mathrm{KdV}$ hierarchies. The first example was given by Pinkall, who in [47] constructed a hierarchy of central affine curve flows on $\mathbb{R}^{2}$ invariant under the group $S L(2, \mathbb{R})$ and showed that their differential invariant (the central affine curvature) satisfies the KdV hierarchy. Calini-Ivey-Mari Beffa in [48] (for $n=3$ ) and Terng and Wu in [49] (for general $n$ ) constructed a hierarchy of curve flows on the affine space $\mathbb{R}^{n}$ invariant under $S L(n, \mathbb{R})$ whose differential invariants satisfy the $\hat{A}_{n-1}^{(1)}-\mathrm{KdV}$ hierarchies. Terng and Wu also constructed in [50] two hierarchies of curve flows on $\mathbb{R}^{n+1, n}$, whose differential invariants under the group $O(n+1, n)$ are solutions of the $\hat{B}_{n}^{(1)}-\mathrm{KdV}$ and $\hat{A}_{2 n}^{(2)}-\mathrm{KdV}$ hierarchies respectively. In this paper, we construct two hierarchies of curve flows on the symplectic space $\mathbb{R}^{2 n}$ whose differential invariants under the symplectic group are solutions of the $\hat{C}_{n}^{(1)}-\mathrm{KdV}$ and the $\hat{A}_{2 n-1}^{(2)}-\mathrm{KdV}$ hierarchies respectively.

We need to set up some more notations before we explain our results. Let $\mathbb{R}^{2 n}$ be the symplectic space with the symplectic form

$$
\omega(X, Y)=X^{t} S_{n} Y, \quad \text { where } S_{n}=\sum_{i=1}^{2 n}(-1)^{i+1} e_{i, 2 n+1-i}
$$

$S p(2 n)=\left\{g \in G L(2 n, \mathbb{R}) \mid g^{t} S_{n} g=S_{n}\right\}$ the group of linear isomorphisms of $\mathbb{R}^{2 n}$ that preserves $w$, and

$$
s p(2 n)=\left\{A \in \operatorname{sl}(2 n) \mid A^{t} S_{n}+S_{n} A=0\right\}
$$

the Lie algebra of $S p(2 n)$. A linear subspace $V$ of $\mathbb{R}^{2 n}$ is isotropic if $\omega(x, y)=0$ for all $x, y \in V$. A maximal isotropic subspace has dimension $n$, and is called Lagrangian. The action of $S p(2 n)$ on the space of Lagrangian subspaces of $\mathbb{R}^{2 n}$ defined by $g \cdot V=g V$ is transitive.

Definition 1. A smooth map $\gamma: \mathbb{R} \rightarrow \mathbb{R}^{2 n}$ is a Lagrangian curve if

(i) $\gamma(s), \gamma_{s}(s), \ldots, \gamma_{s}^{(2 n-1)}(s)$ are linearly independent for all $s \in \mathbb{R}$,

(1) the span of $\gamma(s), \ldots, \gamma_{s}^{(n-1)}(s)$ is a Lagrangian subspace of $\mathbb{R}^{2 n}$ for all $s \in \mathbb{R}$, where $\gamma_{s}^{(i)}=\frac{\mathrm{d}^{i} \gamma}{\mathrm{d} s}$.

We show that if $\gamma: \mathbb{R} \rightarrow \mathbb{R}^{2 n}$ is Lagrangian then there exists a unique orientation preserving parameter $x=x(s)$ such that $\omega\left(\gamma_{x}^{(n)}, \gamma_{x}^{(n-1)}\right)=(-1)^{n}$. We call such parameter the Lagrangian parameter for $\gamma$.

Let

$$
\begin{aligned}
& \mathcal{M}_{2 n}=\left\{\gamma \in \mathbb{R}^{2 n} \mid \gamma \text { is Lagrangian, } \omega\left(\gamma_{s}^{(n)}, \gamma_{s}^{(n-1)}\right)=(-1)^{n}\right\} . \\
& V_{n}=\oplus_{i=1}^{n} \mathbb{R} e_{n+1-i, n+i}, \text { where } \oplus \text { is the direct sum. }
\end{aligned}
$$


We prove that given $\gamma \in \mathcal{M}_{2 n}$, there exists a unique $g=\left(g_{1}, \ldots, g_{2 n}\right): \mathbb{R} \rightarrow S p(2 n)$ such that $g_{i}=\gamma_{x}^{(i-1)}$ for $1 \leq i \leq n+1$ and

$$
g^{-1} g_{x}=b+u
$$

for some $u=\sum_{i=1}^{n} u_{i} e_{n+1-i, n+i} \in C^{\infty}\left(\mathbb{R}, V_{n}\right)$, where

$$
b=\sum_{i=1}^{n-1} e_{i+1, i}
$$

We call this $g$ the Lagrangian moving frame and $u=\sum_{i=1}^{n} u_{i} e_{n+1-i, n+i}$ the Lagrangian curvature along $\gamma$.

It is easy to see that

$$
\gamma(x)=\left(1, x, \frac{x^{2}}{2 !}, \cdots, \frac{x^{2 n-1}}{(2 n-1) !}\right)^{t}
$$

is in $\mathcal{M}_{2 n}$ with Lagrangian frame $g(x)=\exp (b x)$ and zero Lagrangian curvature.

Definition 2. The Lagrangian curvature map

$$
\Psi: \mathcal{M}_{2 n} \rightarrow C^{\infty}\left(\mathbb{R}, V_{n}\right),
$$

is defined by $\Psi(\gamma)=u$, where $u$ is the Lagrangian curvature of $\gamma \in \mathcal{M}_{2 n}$.

It follows from the theory of existence and uniqueness of solutions of ordinary differential equations that the Lagrangian curvatures form a complete set of differential invariants for curves in $\mathcal{M}_{2 n}$.

A Lagrangian curve flow is an evolution equation on $\mathcal{M}_{2 n}$, i.e., the flow preserves the Lagrangian parameter. Such flow can be written in the form $\gamma_{t}=g \xi(u)$ so that $g \xi(u)$ is tangent to $\mathcal{M}_{2 n}$ at $\gamma$, where $g(\cdot, t)$ and $u(\cdot, t)$ are the Lagrangian moving frame and Lagrangian curvature along $\gamma(\cdot, t)$ and $\xi(u)$ is a $\mathbb{R}^{2 n \times 1}$ valued differential polynomial of $u$ in $x$ variable.

Please note that when $n=1$, we have $s p(2)=s l(2, \mathbb{R}), \omega(X, Y)=\operatorname{det}(X, Y)$, the Lagrangian parameter, frame, curvature are the central affine parameter, frame, central affine curvature on $\mathbb{R}^{2}$ under the group $S L(2, \mathbb{R})$, and the Lagrangian curve flows on $\mathbb{R}^{2}$ are the central affine curve flows studied in [47] (see also in [51,52]). For example,

$$
\gamma_{t}=\frac{u_{x}}{4} \gamma-\frac{u}{2} \gamma_{x}
$$

is a Lagrangian flow on $\mathbb{R}^{2}$ and its Lagrangian curvature $u$ satisfies the $\mathrm{KdV}$,

$$
u_{t}=\frac{1}{4}\left(u_{x x x}-6 u u_{x}\right) .
$$

In this paper, we construct two hierarchies of Lagrangian curve flows on $\mathbb{R}^{2 n}$ whose Lagrangian curvatures are solutions of the $\hat{C}_{n}^{(1)}-\mathrm{KdV}$ and $\hat{A}_{2 n-1}^{(2)}-\mathrm{KdV}$ hierarchies respectively. In particular, we obtain the following results:

(1) We construct a sequence of commuting Lagrangian curve flows of C-type and A-type respectively on $\mathcal{M}_{2 n}$ such that the third flows are

$$
\begin{aligned}
& \gamma_{t}=-\frac{3}{4 n}\left(u_{1}\right)_{x} \gamma-\frac{3}{2 n} u_{1} \gamma_{x}+\gamma_{x x x}, \\
& \gamma_{t}=-\frac{3}{2 n-1} u_{1} \gamma_{x}+\gamma_{x x x}
\end{aligned}
$$


respectively, where $u_{1}$ is the first Lagrangian curvature.

(2) The Lagrangian curvature map $\Psi$ maps the space of solutions of Lagrangian curve flows of $C$-type (A-type resp.) modulo $S p(2 n)$ bijectively onto the space of solutions of $\hat{C}_{n}^{(1)}-\mathrm{KdV}\left(\hat{A}_{2 n-1}^{(2)}-\mathrm{KdV}\right.$ resp.) flows. For example, the Lagrangian curvatures $u_{1}, u_{2}$ of a solution $\gamma$ of (3) and (4) satisfy the third $\hat{C}_{2}^{(1)}-\mathrm{KdV}$ flow

$$
\left\{\begin{array}{l}
\left(u_{1}\right)_{t}=-\frac{5}{4} u_{1}^{(3)}+3 u_{2}^{\prime}+\frac{3}{4} u_{1} u_{1}^{\prime} \\
\left(u_{2}\right)_{t}=-\frac{3}{8} u_{1}^{(5)}+u_{2}^{(3)}+\frac{3}{8}\left(u_{1} u_{1}^{(3)}+u_{1}^{\prime} u_{1}^{\prime \prime}\right)-\frac{3}{4} u_{1} u_{2}^{\prime} .
\end{array}\right.
$$

and the third $\hat{A}_{3}^{(2)}-\mathrm{KdV}$ flow

$$
\left\{\begin{array}{l}
\left(u_{1}\right)_{t}=3\left(u_{2}\right)_{x} \\
\left(u_{2}\right)_{t}=\left(u_{2}\right)_{x x x}-\left(u_{1} u_{2}\right)_{x}
\end{array}\right.
$$

respectively.

(3) A bi-Hamiltonian structure and commuting conservation laws for Lagrangian curve flows of C- and A-types are given. For example, the curve flows (3) and (4) are Hamiltonian flows for functionals

$$
\begin{aligned}
\hat{F}_{3}(\gamma) & =\oint u_{2}+\frac{2 n-3}{4 n} u_{1}^{2} \mathrm{~d} x \\
\hat{H}_{3}(\gamma) & =\oint u_{2}+\frac{n-2}{2 n-1} u_{1}^{2} \mathrm{~d} x
\end{aligned}
$$

respectively on $\mathcal{M}_{2 n}$ with respect to the second Hamiltonian structure, where $u$ is the Lagrangian curvature of $\gamma$.

(4) We construct Darboux transforms (DTs), Permutability formulas, scaling transforms, and give an algorithm to compute explicit soliton solutions of these flows.

This paper is organized as follows: We construct Lagrangian moving frames in Section 2, and review the constructions of the $\hat{C}_{n}^{(1)}-\mathrm{KdV}$ and $\hat{A}_{2 n-1}^{(2)}-\mathrm{KdV}$ hierarchies in Section 3. Lagrangian curve flows of $C$ - and $A$ - types and the evolutions of their Lagrangian curvatures are given in Section 4. In Section 5, we construct Darboux transforms (DTs) and a Permutability formula for the $\hat{C}_{n}^{(1)}-\mathrm{KdV}$ and for the Lagrangian curve flows of C-type. DTs for the A case and its Permutability formula are given in Section 6. The scaling transforms are given in Section 7. Bi-Hamiltonian structures and commuting conserved functionals are given in Section 8. We give an outline of a method for constructing integrable curve flows whose differential invariants satisfy the $\hat{\mathcal{G}}^{(1)}-\mathrm{KdV}$ hierarchy for general simple real non-compact Lie algebra $\mathcal{G}$ and give some open problems in the last section.

\section{Lagrangian Moving Frame}

In this section, we prove the existence of Lagrangian parameter and construct the Lagrangian moving frame and curvatures for Lagrangian curves (cf. Definition 1).

Proposition 1. If $\gamma: \mathbb{R} \rightarrow \mathbb{R}^{2 n}$ is a Lagrangian curve, then there exists a unique Lagrangian parameter $x=x(s)$, i.e., $\omega\left(\gamma_{x}^{(n)}, \gamma_{x}^{(n-1)}\right)=(-1)^{n}$.

Proof. If $\omega\left(\gamma_{s}^{(n)}, \gamma_{s}^{(n-1)}\right)$ is zero at $s_{0}$, then it follows from $\omega\left(\gamma_{s}^{(i)}, \gamma_{s}^{(j)}\right)=0$ for all $0 \leq$ $i, j \leq n-1$ that $\omega\left(\gamma_{s}^{(n)}, \gamma_{s}^{(i)}\right)=0$ at $s_{0}$. Hence $\gamma\left(s_{0}\right), \gamma_{s}\left(s_{0}\right), \ldots, \gamma_{s}^{(n)}\left(s_{0}\right)$ span an $(n+1)$ dimension isotropic subspace. However, the maximal dimension of an isotropic subspace is $n$, a contradiction. Hence $\omega\left(\gamma_{s}^{(n)}, \gamma_{s}^{(n-1))}\right)$ never vanishes. Choose $x=x(s)$ such that $\left(\frac{\mathrm{d} x}{\mathrm{~d} s}\right)^{2 n-1}=(-1)^{n} \omega\left(\gamma_{s}^{(n)}, \gamma_{s}^{(n-1)}\right)$. 
Theorem 1. If $\gamma \in \mathcal{M}_{2 n}$, then there exists a unique Lagrangian moving frame $g$ along $\gamma$, i.e., $g^{-1} g_{x}=b+\sum_{i=1}^{n} u_{i} e_{n+1-i, n+i}$ for some $u_{1}, \ldots, u_{n}$, where $b$ is defined by (2).

Proof. Let $u_{1}=(-1)^{n-1} \omega\left(\gamma_{x}^{(n+1)}, \gamma_{x}^{(n)}\right)$, and $g_{n+2}=\gamma_{x}^{(n+1)}-u_{1} \gamma_{x}^{(n-1)}$. We derive $g_{i}{ }^{\prime} \mathrm{s}$ and $u_{i}$ 's by the recursive formula:

$$
\begin{aligned}
& u_{j}=(-1)^{n-j} \omega\left(\left(g_{n+j}\right)_{x}, g_{n+j}\right)=(-1)^{n-j} \omega\left(\mathrm{d}_{x}^{n+j} \gamma, g_{n+j}\right), \quad 2 \leq j \leq n-1, \\
& g_{n+j+1}=\mathrm{d}_{x} g_{n+j}-u_{j} \gamma_{x}^{(n-j)}, \quad 2 \leq j \leq n-1, \\
& u_{n}=\omega\left(\left(g_{2 n}\right)_{x}, g_{2 n}\right) .
\end{aligned}
$$

Then $g=\left(\gamma, \ldots, \gamma_{x}^{(n)}, g_{n+2}, \ldots, g_{2 n}\right)$ satisfies $g^{-1} g_{x}=b+u$, i.e., $g$ is a Lagrangian moving frame along $\gamma$.

Example 1. For $n=1$, we have $\omega(X, Y)=\operatorname{det}(X, Y)$, thus $\gamma \in \mathcal{M}_{2}$ if and only if $\operatorname{det}\left(\gamma, \gamma_{x}\right)=$ 1. So the Lagrangian parameter is the central affine parameter, the Lagrangian frame along $\gamma$ is $g=\left(\gamma, \gamma_{x}\right)$ is the central affine moving frame along $\gamma$, and the Lagrangian curvature is the central affine curvature. Moreover,

$$
g^{-1} g_{x}=\left(\begin{array}{cc}
0 & u_{1} \\
1 & 0
\end{array}\right)
$$

Example 2. The Lagrangian frame $g=\left(\gamma, \gamma_{x}, \gamma_{x x}, g_{4}\right)$ along $\gamma \in \mathcal{M}_{4}$ satisfies

$$
g^{-1} g_{x}=\left(\begin{array}{cccc}
0 & 0 & 0 & u_{2} \\
1 & 0 & u_{1} & 0 \\
0 & 1 & 0 & 0 \\
0 & 0 & 1 & 0
\end{array}\right)
$$

where

$$
u_{1}=-\omega\left(\gamma_{x}^{(3)}, \gamma_{x x}\right), \quad u_{2}=\omega\left(\left(g_{4}\right)_{x}, g_{4}\right)=\omega\left(\gamma_{x}^{(4)}, \gamma\right), \quad g_{4}=\gamma_{x}^{(3)}-u_{1} \gamma_{x}
$$

It follows from the Existence and Uniqueness of ordinary differential equations that $\left\{u_{1}, \cdots, u_{n}\right\}$ forms a complete set of local differential invariants for $\gamma \in \mathcal{M}_{2 n}$ under the $S p(2 n)$-action. So we have the following:

Proposition 2. The Lagrangian curvature map $\Psi: \mathcal{M}_{2 n} \rightarrow C^{\infty}\left(\mathbb{R}, V_{n}\right)$ defined by Definition 2 is onto and $\Psi^{-1}(u)$ is a $\operatorname{Sp}(2 n)$-orbit.

Example 3. A Lagrangian curve in $\mathbb{R}^{2 n}$ with zero Lagrangian curvature is of the form:

$$
\gamma=c_{0}\left(1, x, \frac{x^{2}}{2}, \cdots, \frac{x^{2 n-1}}{(2 n-1) !}\right)^{t}, \quad c_{0} \in S p(2 n) .
$$

\section{The $\hat{C}_{n}^{(1)}-\mathrm{KdV}$ and the $\hat{A}_{2 n-1}^{(2)}-\mathrm{KdV}$ Hierarchies}

In this section, we review the constructions of the $\hat{C}_{n}^{(1)}{ }^{-}, \hat{A}_{2 n-1^{-}}^{(2)} \hat{C}_{n}^{(1)}-\mathrm{KdV}$, and $\hat{A}_{2 n-1^{-}}^{(2)}$ $\mathrm{KdV}$ hierarchies and derive some elementary properties of these hierarchies (cf. [11,53]).

3.1. The $\hat{C}_{n}^{(1)}-K d V$ Hierarchy

A splitting of a Lie algebra $\mathcal{L}$ is a pair of Lie subalgebras $\mathcal{L}_{+}, \mathcal{L}_{-}$such that $\mathcal{L}=$ $\mathcal{L}_{+} \oplus \mathcal{L}_{-}$as linear subspaces (but not as subalgebras). For $\xi \in \mathcal{L}$, we write

$$
\xi=\xi_{+}+\xi_{-}, \quad \text { where } \xi_{+} \in \mathcal{L}_{+}, \xi_{-} \in \mathcal{L}_{-} .
$$


A vacuum sequence is a linearly independent, commuting sequence $\left\{J_{j} \mid j \geq 1\right\}$ in $\mathcal{L}_{+}$. Let

$$
\operatorname{Sp}(2 n, \mathbb{C})=\left\{A \in G L(2 n, \mathbb{C}) \mid A^{t} S_{n} A=S_{n}\right\},
$$

and $\operatorname{sp}(2 n, \mathbb{C})$ its Lie algebra. Then $s p(2 n)$ is a real form of $s p(2 n, \mathbb{C})$ defined by the involution $\tau(A)=\bar{A}$.

Let

$$
\begin{aligned}
& \hat{C}_{n}^{(1)}:=\left\{A=\sum_{i} A_{i} \lambda^{i} \mid A_{i} \in \operatorname{sp}(2 n)\right\}, \\
& \left(\hat{C}_{n}^{(1)}\right)_{+}=\left\{\sum_{i \geq 0} A_{i} \lambda^{i} \in \hat{C}_{n}^{(1)}\right\}, \quad\left(\hat{C}_{n}^{(1)}\right)_{-}=\left\{\sum_{i<0} A_{i} \lambda^{i} \in \hat{C}_{n}^{(1)}\right\} .
\end{aligned}
$$

Then $\left(\left(\hat{C}_{n}^{(1)}\right)_{+},\left(\hat{C}_{n}^{(1)}\right)_{-}\right)$is a splitting of $\hat{C}_{n}^{(1)}$.

Please note that $\xi(\lambda)=\sum_{i} \xi_{i} \lambda^{i}$ is in $\hat{C}_{n}^{(1)}$ if and only if $\xi$ satisfy the $s p(2 n)$-reality condition, i.e.,

$$
\xi(\lambda)^{t} S_{n}+S_{n} \xi(\lambda)=0, \quad \overline{\xi(\bar{\lambda})}=\xi(\lambda) .
$$

A meromorphic map $f: \mathbb{C} \rightarrow S L(2 n, \mathbb{C})$ is said to satisfy the $S p(2 n)$-reality condition if

$$
f(\lambda)^{t} S_{n} f(\lambda)=S_{n}, \quad \overline{f(\bar{\lambda})}=f(\lambda)
$$

For $\xi(\lambda)=\sum_{i} \xi_{i} \lambda^{i}$, we have

$$
\xi_{+}(\lambda)=\sum_{i \geq 0} \xi_{i} \lambda^{i}, \quad \xi_{-}(\lambda)=\sum_{i<0} \xi_{i} \lambda^{i}
$$

Let $B_{n}^{+}$and $N_{n}^{+}$denote the subgroups of upper, strictly upper triangular matrices in $S p(2 n)$ respectively, and $\mathcal{B}_{n}^{+}, \mathcal{N}_{n}^{+}$the corresponding Lie subalgebras of $s p(2 n)$.

Set

$$
J=\sum_{i=1}^{2 n-1} e_{i+1, i}+e_{1,2 n} \lambda=b+e_{1,2 n} \lambda \in\left(\hat{C}_{n}^{(1)}\right)_{+} .
$$

Then

$$
\begin{aligned}
J^{i} & =\left(b^{t}\right)^{n-i} \lambda+b^{i}, \quad 1 \leq i \leq 2 n-1, \\
J^{2 n} & =\lambda I_{2 n} .
\end{aligned}
$$

It is easy to check that $J^{2 j-1}$ is in $\left(\hat{C}_{n}^{(1)}\right)_{+}$, but $J^{2 j}$ is not. So $\left\{J^{2 j-1} \mid j \geq 1\right\}$ is a vacuum sequence. Note that

$$
\left[J,\left(\hat{C}_{n}^{(1)}\right)_{-}\right]_{+}=\mathcal{B}_{n}^{+}
$$

Next we use the general method given in [53] to construct the $\hat{C}_{n}^{(1)}$-hierarchy generated by the vacuum sequence $\left\{J^{2 j-1} \mid j \geq 1\right\}$. First a direct computation gives the following known results:

Theorem $2([49,53])$. Given $q \in C^{\infty}\left(\mathbb{R}, \mathcal{B}_{n}^{+}\right)$, then there exists a unique

$$
P(q, \lambda)=\sum_{i \leq 1} P_{1, i}(q) \lambda^{i}
$$

in $\hat{C}_{n}^{(1)}$ satisfying

$$
\left\{\begin{array}{l}
{\left[\partial_{x}+J+q, P(q, \lambda)\right]=0} \\
P^{2 n}(q, \lambda)=\lambda \mathrm{I}_{2 n}
\end{array}\right.
$$


Moreover, $P_{1, i}(q)$ can be computed recursively by equating the coefficients of $\lambda^{i}$ in (10) and they are polynomials in $u$ and $x$-derivatives of $u$ (i.e., a differential polynomial in $u$ ).

Please note that if operators $A, B$ commute, then $A$ and $B^{j}$ also commute. Hence it follows from the first equation of (10) that we have

$$
\left[\partial_{x}+J+q, P^{2 j-1}(q, \lambda)\right]=0
$$

Write the power series

$$
P^{2 j-1}(q, \lambda)=\sum_{i} P_{2 j-1, i}(q) \lambda^{i}
$$

We compare coefficient of $\lambda^{i}$ of (11) to obtain

$$
\left[\partial_{x}+b+q, P_{2 j-1, i}(q)\right]=\left[P_{2 j-1, i-1}(q), e_{1,2 n}\right],
$$

which implies that the left hand side lies in $\mathcal{B}_{n}^{+}$. So

$$
q_{t_{2 j-1}}=\left[\partial_{x}+b+q, P_{2 j-1,0}(q)\right], \quad j \geq 1 \text {. }
$$

defines a flow on $C^{\infty}\left(\mathbb{R}, \mathcal{B}_{n}^{+}\right)$. We call (14) the $(2 j-1)$-th $\hat{C}_{n}^{(1)}$-flow.

We need the following well-known elementary result to explain the Lax pair:

Proposition 3. Let $\mathcal{G}$ be the Lie algebra of $G$, and $A, B: \mathbb{R}^{2} \rightarrow \mathcal{G}$ smooth maps. Then the following statements are equivalent:

(1) the linear system

$$
\left\{\begin{array}{l}
g_{x}=g A, \\
g_{t}=g B
\end{array}\right.
$$

is solvable for $g: \mathbb{R}^{2} \rightarrow G$,

(2) A, B satisfy

$$
A_{t}=B_{x}+[A, B]=\left[\partial_{x}+A, B\right]
$$

(3) $\left[\partial_{x}+A, \partial_{t}+B\right]=0$.

Proposition 4. The following statements are equivalent for smooth $q: \mathbb{R}^{2} \rightarrow \mathcal{B}_{n}^{+}$:

(1) $q$ is a solution of (14),

(2) the following linear system is solvable for $h: \mathbb{R}^{2} \rightarrow S p(2 n)$,

$$
\left\{\begin{array}{l}
h^{-1} h_{x}=b+q, \\
h^{-1} h_{t}=P_{2 j-1,0}(q) .
\end{array}\right.
$$

(3) the following linear system is solvable for $F(x, t, \lambda) \in S L(2 n, \mathbb{C})$,

$$
\left\{\begin{array}{l}
F_{x}=F(J+q), \\
F_{t}=F\left(P^{2 j-1}(q, \lambda)\right)_{+}, \\
F(x, t, \lambda)^{t} S_{n} F(x, t, \lambda)=S_{n}, \quad \overline{F(x, t, \bar{\lambda})}=F(x, t, \lambda) .
\end{array}\right.
$$

The last equation says that $F(x, t, \lambda)$ satisfies the $S p(2 n)$-reality condition (7) in $\lambda$.

Proof. Equation (13) implies that the coefficients of $\lambda^{i}$ for $i>0$ of

$$
\left[\partial_{x}+J+q, \partial_{t}+\left(P^{2 j-1}(q, \lambda)\right)_{+}\right]
$$


are zero. The constant term is $\left[\partial_{x}+b+q, \partial_{t}+P_{2 j-1,0}(q)\right]$. This proves that $\left[\partial_{x}+J+\right.$ $\left.q, \partial_{t}+\left(P^{2 j-1}(q, \lambda)\right)_{+}\right]=0$ is equivalent to $\left[\partial_{x}+b+q, \partial_{t}+P_{2 j-1,0}(q)\right]=0$. It follows from Proposition 3 that (2) and (3) are equivalent.

Equation (14) can be written as

$$
(b+q)_{t}=\left(P_{2 j-1,0}(q)\right)_{x}+\left[b+q, P_{2 j-1,0}(q)\right] .
$$

It follows from Proposition 3 that (1) and (2) are equivalent.

The group $C^{\infty}\left(\mathbb{R}, N_{n}^{+}\right)$acts on $C^{\infty}\left(\mathbb{R}, \mathcal{B}_{n}^{+}\right)$by gauge transformation,

$$
f\left(\partial_{x}+b+q\right) f^{-1}=\partial_{x}+b+f * q
$$

for $f \in C^{\infty}\left(\mathbb{R}, N_{n}^{+}\right)$and $q \in C^{\infty}\left(\mathbb{R}, \mathcal{B}_{n}^{+}\right)$, where

$$
f * q=f(b+q) f^{-1}-f_{x} f^{-1}-b .
$$

The following Proposition shows that $C^{\infty}\left(\mathbb{R}, V_{n}\right)$ is a cross-section of this gauge action.

Proposition 5. Given $q \in C^{\infty}\left(\mathbb{R}, \mathcal{B}_{n}^{+}\right)$, then there exist a unique $\triangle \in C^{\infty}\left(\mathbb{R}, N_{n}^{+}\right)$and $u=$ $\sum_{i=1}^{n} u_{i} e_{n+1-i, n+i}$ in $C^{\infty}\left(\mathbb{R}, V_{n}\right)$ such that

$$
\triangle\left(\partial_{x}+J+q\right) \triangle^{-1}=\partial_{x}+J+u .
$$

In particular, $u=\triangle * q$.

Proof. Let $\mathcal{G}_{j}=\oplus_{i=1}^{2 n-j} \mathbb{R} e_{i, i+j}, \mathcal{G}_{-j}=\oplus_{i=1}^{2 n-j} \mathbb{R} e_{i+j, i}$ for $0 \leq j \leq 2 n-1$. Equation (19) implies that

$$
\triangle(J+q)-\triangle_{x}=(J+u) \triangle .
$$

Proposition is proved by equating components of $\mathcal{G}_{j}$ of (20) for $|j| \leq 2 n-1$.

It can be checked by the same method for the $\hat{A}_{n}^{(1)}$-hierarchy (cf. [53]) that flow (14) is invariant under the $C^{\infty}\left(\mathbb{R}, N_{n}^{+}\right)$-action. So given $u \in C^{\infty}\left(\mathbb{R}, V_{n}\right)$ and $j \geq 1$, there exists a unique $\mathcal{N}_{n}^{+}$-valued differential polynomial $\eta_{j}(u)$ satisfying

$$
\left[\partial_{x}+J+u,\left(P(u)^{2 j-1}\right)_{+}-\eta_{j}(u)\right] \in C^{\infty}\left(\mathbb{R}, V_{n}\right) .
$$

The induced quotient flow of (14) on the cross-section $C^{\infty}\left(\mathbb{R}, V_{n}\right)$ is obtained by projecting (14) down along gauge orbits. So the induced quotient flow on $C^{\infty}\left(\mathbb{R}, V_{n}\right)$ is

$$
u_{t_{2 j-1}}=\left[\partial+J+u, P_{2 j-1,0}(u)-\eta_{j}(u)\right] .
$$

The above equation is the $(2 j-1)-t h \hat{C}_{n}^{(1)}-K d V$ flow.

As a consequence of the construction, we have the following.

Proposition 6. The following statements are equivalent for smooth $u: \mathbb{R}^{2} \rightarrow V_{n}$ :

(i) $u$ is a solution of (22),

(ii) $\left[\partial_{x}+J+u, \partial_{t}+\left(P^{2 j-1}(u, \lambda)\right)_{+}-\eta_{j}(u)\right]=0$.

(iii) The following linear system is solvable for $g: \mathbb{R}^{2} \rightarrow S p(2 n)$,

$$
\left\{\begin{array}{l}
g^{-1} g_{x}=b+u, \\
g^{-1} g_{t}=P_{2 j-1,0}(u)-\eta_{j}(u) .
\end{array}\right.
$$


(iv) The following linear system is solvable for $E(x, t, \lambda) \in S L(2 n, \mathbb{C})$ for all parameter $\lambda \in \mathbb{C}$,

$$
\left\{\begin{array}{l}
E_{x}=E(J+u) \\
E_{t}=E\left(\left(P^{2 j-1}(u, \lambda)\right)_{+}-\eta_{j}(u)\right) \\
E(x, t, \lambda)^{t} S_{n} E(x, t, \lambda)=S_{n}, \quad \overline{E(x, t, \bar{\lambda})}=E(x, t, \lambda) .
\end{array}\right.
$$

Example 4. The third $\hat{C}_{1}^{(1)}-K d V$ flow is the $K d V$ for $q=u_{1}$ :

$$
q_{t}=\frac{1}{4}\left(q_{x x x}-6 q q_{x}\right)
$$

\section{Example 5. The third $\hat{C}_{2}^{(1)}-\mathrm{KdV}$ flow}

The $\hat{C}_{2}^{(1)}-K d V$ flows are for $u=u_{1} e_{23}+u_{2} e_{14}$. We compare coefficients of $\lambda^{i}$ in (10) to compute $P_{1, i}(q)$. Then $P_{2 j-1, i}(q)$ can be computed from $P_{1, k}(q)$ 's. We obtain the first few terms of $P^{3}(q, \lambda)$ :

$$
\begin{aligned}
& P_{3,1}(u)=\left(\begin{array}{cccc}
0 & 1 & 0 & -\frac{3}{4} u_{1} \\
0 & 0 & 1 & 0 \\
0 & 0 & 0 & 1 \\
0 & 0 & 0 & 0
\end{array}\right), \\
& P_{3,0}(u)=\left(\begin{array}{cccc}
-\frac{3}{8} u_{1}^{\prime} & u_{2}-\frac{3}{8} u_{1}^{\prime \prime} & u_{2}^{\prime}-\frac{3}{8}\left(u_{1}\right)_{x}^{(3)} & \xi \\
\frac{1}{4} u_{1} & -\frac{1}{8} u_{1}^{\prime} & -\frac{1}{2} u_{1}^{\prime \prime}+u_{2}+\frac{1}{4} u_{1}^{2} & -u_{2}^{\prime}+\frac{3}{8}\left(u_{1}\right)_{x}^{(3)} \\
0 & \frac{1}{4} u_{1} & \frac{1}{8} u_{1}^{\prime} & u_{2}-\frac{3}{8} u_{1}^{\prime \prime} \\
1 & 0 & \frac{1}{4} u_{1} & \frac{3}{8} u_{1}^{\prime}
\end{array}\right), \text { where } \\
& \xi=-\frac{3}{8}\left(u_{1}\right)_{x}^{(4)}+u_{2}^{\prime \prime}+\frac{3}{8} u_{1} u_{1}^{\prime \prime}-\frac{3}{4} u_{1} u_{2} .
\end{aligned}
$$

Therefore the third $\hat{C}_{2}^{(1)}-K d V$ flow is (5).

3.2. The $\hat{A}_{2 n-1}^{(2)}-K d V$ Hierarchy $(n \geq 2)$

Let $\kappa$ be the involution of $s l(2 n, \mathbb{C})$ defined by

$$
\kappa(X)=-S_{n} X^{t} S_{n}^{-1}
$$

where $S_{n}$ is as in (1). Then the fixed point set of $\kappa$ is $s p(2 n, \mathbb{C})$ and the -1 eigen-space of $\kappa$ in $\operatorname{sl}(2 n)$ is

$$
\mathcal{P}_{2 n}=\left\{\xi \in \operatorname{sl}(2 n) \mid \xi=S_{n} \xi^{t} S_{n}^{-1}\right\}
$$

Let

$$
\hat{A}_{2 n-1}^{(2)}=\left\{A(\lambda)=\sum_{i \leq m_{0}} A_{i} \lambda^{i} \mid A_{i} \in \operatorname{sl}(2 n, \mathbb{R}), \kappa(A(-\lambda))=A(\lambda)\right\}
$$

and

$$
\left(\hat{A}_{2 n-1}^{(2)}\right)_{+}=\left\{\sum_{i \geq 0} A_{i} \lambda^{i} \in \hat{A}_{2 n-1}^{(2)}\right\}, \quad\left(\hat{A}_{2 n-1}^{(2)}\right)_{-}=\left\{\sum_{i<0} A_{i} \lambda^{i} \in \hat{A}_{2 n-1}^{(2)}\right\} .
$$

Then $\left(\left(\hat{A}_{2 n-1}^{(2)}\right)_{+},\left(\hat{A}_{2 n-1}^{(2)}\right)_{-}\right)$is a splitting of $\hat{A}_{2 n-1}^{(2)}$.

Please note that the following are equivalent for $A(\lambda)=\sum_{i} A_{i} \lambda^{i}$ :

(1) $A \in \hat{A}_{2 n-1}^{(2)}$,

(2) $A_{2 i} \in \operatorname{sp}(2 n)$ and $A_{2 i+1} \in \mathcal{P}_{2 n}$ for all $i$, 
(3) A satisfies

$$
-S_{n} A(-\lambda)^{t} S_{n}^{-1}=A(\lambda), \quad \overline{A(\bar{\lambda})}=A(\lambda) .
$$

Set

$$
\begin{gathered}
\beta=\frac{1}{2}\left(e_{1,2 n-1}+e_{2,2 n}\right) . \\
J_{B}=\frac{1}{2}\left(e_{1,2 n-1}+e_{2,2 n}\right) \lambda+\sum_{i=1}^{2 n-1} e_{i+1, i}=\beta \lambda+b .
\end{gathered}
$$

Please note that $J_{B}^{2 i-1} \in\left(\hat{A}_{2 n-1}^{(2)}\right)_{+}$and

$$
J_{B}^{2 n}=\lambda J_{B}
$$

Then $\left\{J_{B}^{2 j-1} \mid j \geq 1\right\}$ is a vacuum sequence in $\left(\hat{A}_{2 n-1}^{(2)}\right)_{+}$.

Next we use the general method given in [53] to construct the $\hat{A}_{2 n-1}^{(2)}$-hierarchy generated by $\left\{J_{B}^{2 j-1} \mid j \geq 1\right\}$. Similarly, we have the following:

Theorem 3. Given $q \in C^{\infty}\left(\mathbb{R}, \mathcal{B}_{n}^{+}\right)$and $j \in \mathbb{Z}$, then there exists a unique

$$
Q(q, \lambda)=\sum_{i \leq 1} Q_{1, i}(q) \lambda^{i} \in \hat{A}_{2 n-1}^{(2)}
$$

satisfying

$$
\left\{\begin{array}{l}
{\left[\partial_{x}+J_{B}+q, Q(q, \lambda)\right]=0,} \\
Q^{2 n}(q, \lambda)=\lambda Q(q, \lambda)
\end{array}\right.
$$

Moreover, $Q_{1, i}(q)$ 's are polynomial differentials in $q$ and derivatives of $q$ and can be computed recursively by equating the coefficient of $\lambda^{i}$ of (26).

Proof. It was proved in [11] that given any $\xi=\sum_{i \leq i_{0}} \xi_{i} \lambda^{i} \in \operatorname{sl}(2 n, \mathbb{R})$, there exists unique diagonal matrices $h_{i}$ such that

$$
\xi=\sum_{j \leq 2 n\left(i_{0}+1\right)} h_{i} J^{j}
$$

where $J=b+e_{1,2 n} \lambda$.

Given permutation $s$ in $S_{2 n}$ and $h=\operatorname{diag}\left(h_{1}, \ldots, h_{2 n}\right)$, let

$$
h^{\varsigma}=\operatorname{diag}\left(h_{s(1)}, \ldots, h_{s(2 n)}\right) .
$$

Let $\theta \in S_{2 n}$ be the cyclic permutation defined by $\theta(1)=2 n$, and $\theta(i)=i-1$ for $2 \leq i \leq 2 n$. A simple computation implies that

$$
J h=h^{\theta} J, \quad J^{i} h=h^{\theta^{i}} J^{i} .
$$

Please note that

$$
\begin{aligned}
& \frac{1}{2}\left(e_{1,2 n-1}+e_{2 n}\right)=\operatorname{diag}\left(\frac{1}{2}, \frac{1}{2}, 0, \ldots, 0\right) J^{2} . \\
& q=\sum_{i=1}^{2 n} k_{i} J^{i-2 n},
\end{aligned}
$$

where $q=\left(q_{i j}\right)$ and $k_{i}$ are diagonal matrices defined by

$$
k_{i}=\operatorname{diag}\left(q_{1,2 n-i+1}, q_{2,2 n-i+2}, \ldots, q_{i, 2 n}, 0, \ldots, 0\right) .
$$


Write

$$
Q(q, \lambda)=\operatorname{diag}\left(\frac{1}{2}, \frac{1}{2}, 0, \ldots, 0\right) J^{2}+\operatorname{diag}(0,1, \ldots, 1) J+\sum_{j \leq 0} h_{j} J^{j}
$$

We compare the coefficients of $J^{j}$ 's of both sides of each equation in (26) and use (27) to solve $h_{j}$ uniquely as differential polynomial of $q$. This gives the formula for $Q(q, \lambda)$. We plug in Formulas (8) and (9) to obtain $Q_{1, j}(q)^{\prime}$ s.

The first equation of (26) implies that

$$
\left[\partial_{x}+J_{B}+q, Q^{2 j-1}(q, \lambda)\right]=0 .
$$

Write $Q^{2 j-1}(q, \lambda)$ as a power series in $\lambda$,

$$
Q^{2 j-1}(q, \lambda)=\sum_{i} Q_{2 j-1, i}(q) \lambda^{i}
$$

We compare the coefficient of $\lambda^{i}$ of (28) to obtain

$$
\left[\partial_{x}+b+q, Q_{2 j-1, i}(q)\right]=\left[Q_{2 j-1, i-1}(q), \beta\right],
$$

where $\beta$ is defined by (25). So the left hand side of (30) is $\mathcal{B}_{n}^{+}$-valued and

$$
q_{t_{2 j-1}}=\left[\partial_{x}+b+q, Q_{2 j-1,0}(q)\right], \quad j \geq 1
$$

is a flow on $C^{\infty}\left(\mathbb{R}, \mathcal{B}_{n}^{+}\right)$. This is the $(2 j-1)$-th flow in the $\hat{A}_{2 n-1}^{(2)}$-hierarchy.

We use the same proof of Proposition 4 to obtain the following:

Proposition 7. The following statements are equivalent for smooth $q: \mathbb{R}^{2} \rightarrow \mathcal{B}_{n}^{+}$:

(i) $q$ is a solution of (31).

(ii) The following linear system is solvable for smooth $g: \mathbb{R}^{2} \rightarrow S p(2 n)$,

$$
\left\{\begin{array}{l}
g^{-1} g_{x}=b+q \\
g^{-1} g_{t}=Q_{2 j-1,0}(u)
\end{array}\right.
$$

(iii) The following linear system is solvable for $F(x, t, \lambda) \in S L(2 n, \mathbb{C})$ for all parameter $\lambda \in \mathbb{C}$,

$$
\left\{\begin{array}{l}
F_{x}=F\left(J_{B}+q\right) \\
F_{t}=F\left(\left(Q_{2 j-1}(u, \lambda)\right)_{+}\right. \\
F(x, t, \lambda)^{t} S_{n} F(x, t,-\lambda)=S_{n}, \quad \overline{F(x, t, \bar{\lambda})}=F(x, t, \lambda) .
\end{array}\right.
$$

It follows from Proposition 5 that there exist a unique $\triangle \in C^{\infty}\left(\mathbb{R}, N_{n}^{+}\right)$and $u \in$ $C^{\infty}\left(\mathbb{R}, V_{n}\right)$ such that $u=\triangle * q$. So given $u \in C^{\infty}\left(\mathbb{R}, V_{n}\right)$ and $j \geq 1$, there exists a unique $\xi_{j}(u) \in C^{\infty}\left(\mathbb{R}, \mathcal{N}_{n}^{+}\right)$such that

$$
\left[\partial_{x}+b+u, Q_{2 j-1,0}(u)-\xi_{j}(u)\right] \in C^{\infty}\left(\mathbb{R}, V_{n}\right)
$$

The $(2 j-1)-t h \hat{A}_{2 n-1}^{(2)}-K d V$ flow is the following flow on $C^{\infty}\left(\mathbb{R}, V_{n}\right)$ :

$$
u_{t_{2 j-1}}=\left[\partial_{x}+b+u, Q_{2 j-1,0}(u)-\xi_{j}(u)\right]
$$

Proposition 8. The following statements are equivalent for smooth $u: \mathbb{R}^{2} \rightarrow V_{n}$ :

(i) $u$ is a solution of (34). 
(ii) The following linear system is solvable for $g: \mathbb{R}^{2} \rightarrow S p(2 n)$,

$$
\left\{\begin{array}{l}
g^{-1} g_{x}=b+u, \\
g^{-1} g_{t}=Q_{2 j-1,0}(u)-\xi_{j}(u) .
\end{array}\right.
$$

(iii) The following linear system is solvable for $E(x, t, \lambda) \in S L(2 n, \mathbb{C})$ for all $\lambda \in \mathbb{C}$,

$$
\left\{\begin{array}{l}
E_{x}=E\left(J_{B}+u\right) \\
\left.E_{t}=E\left(Q_{2 j-1}(u, \lambda)\right)_{+}-\xi_{j}(u)\right) \\
E(x, t, \lambda)^{t} S_{n} E(x, t,-\lambda)=S_{n}, \overline{E(x, t, \bar{\lambda})}=E(x, t, \lambda) .
\end{array}\right.
$$

Example 6. We use (26) to compute $Q_{1, i}(u)$, then use these to compute $Q_{3,0}(u)$. A direct computation implies that the third $\hat{A}_{3}^{(2)}-K d V$ flow is (6).

Definition 3. $F(x, t, \lambda)\left(E(x, t, \lambda)\right.$ resp.) is a frame of a solution $q: \mathbb{R}^{2} \rightarrow \mathcal{B}_{n}^{+}$of $(14)\left(u: \mathbb{R}^{2} \rightarrow\right.$ $V_{n}$ of (22) resp.) if $F(x, t, \lambda)(E(x, t, \lambda)$ resp.) is holomorphic for all $\lambda \in \mathbb{C}$ and satisfies the linear system (16) ((24) resp.). Frames for solutions of (31) and (34) are defined similarly.

It follows from the constructions of the $\hat{C}_{n}^{(1)}-\mathrm{KdV}$ and $\hat{A}_{2 n-1}^{(2)}-\mathrm{KdV}$ flows that we have the following.

\section{Proposition 9.}

(1) Let $F(x, t, \lambda)$ be a frame of a solution $q: \mathbb{R}^{2} \rightarrow \mathcal{B}_{n}^{+}$of (14) ((31) resp.), and the unique $\triangle: \mathbb{R}^{2} \rightarrow N_{n}^{+}$such that $u:=\triangle * q$ is $V_{n}$-valued (as in Proposition 5). Then $u$ is a solution of (22) ((34) resp.) and $E(x, t, \lambda)=F(x, t, \lambda) \triangle^{-1}(x, t)$ is a frame of $u$, where $*$ is the gauge action defined by (18) or equivalently (17).

(2) Let $E$ be a solution $u: \mathbb{R}^{2} \rightarrow V_{n}$ of (22) ((34) resp.), and $\triangle: \mathbb{R}^{2} \rightarrow N_{n}^{+}$satisfying $\triangle_{t} \triangle^{-1}=\eta_{j}(u)\left(\triangle_{t} \triangle^{-1}=\xi_{j}(u)\right.$ resp. $)$, where $\eta_{j}(u)\left(\xi_{j}(u)\right.$ resp. $)$ is defined by (21) ( (33) resp.). Then $q:=\triangle^{-1} * u$ is a solution of (14) ((31) resp.) and $F=E \triangle$ is a frame of $q$.

\section{Lagrangian Curve Flows on $\mathbb{R}^{2 n}$}

In this section, we

(i) give a description of the tangent space of $\mathcal{M}_{2 n}$ at $\gamma$ and show that it is isomorphic to $C^{\infty}\left(\mathbb{R}, \mathbb{R}^{n}\right)$,

(ii) construct two hierarchies of Lagrangian curve flows whose curvatures satisfy the $\hat{C}_{n}^{(1)}-\mathrm{KdV}$ and the $\hat{A}_{2 n-1}^{(2)}-\mathrm{KdV}$ flows respectively.

Henceforth in this paper we set

$$
e_{1}=(1,0, \ldots, 0)^{t} \in \mathbb{R}^{2 n} .
$$

Theorem 4. Let $g$ and $u$ denote the Lagrangian frame and Lagrangian curvature along $\gamma \in \mathcal{M}_{2 n}$, and $\Psi: \mathcal{M}_{2 n} \rightarrow C^{\infty}\left(\mathbb{R}, V_{n}\right)$ the Lagrangian curvature map. Then

$$
\mathrm{d} \Psi_{\gamma}(\delta \gamma)=\left[\partial_{x}+b+u, g^{-1} \delta g\right]
$$

where $b=\sum_{i=1}^{2 n-1} e_{i+1, i}$. Moreover,

(1) $\quad C: \mathbb{R} \rightarrow \operatorname{sp}(2 n)$ satisfies

$$
\left[\partial_{x}+b+u, C\right] \in C^{\infty}\left(\mathbb{R}, V_{n}\right) .
$$

if and only if $g C e_{1}$ is tangent to $\mathcal{M}_{2 n}$ at $\gamma$,

(2) if $\xi$ is tangent to $\mathcal{M}_{2 n}$ at $\gamma$ then there exists a unique smooth $C: \mathbb{R} \rightarrow \operatorname{sp}(2 n)$ satisfying (37) such that $\xi=g C e_{1}$. 
Proof. A direct computation gives (36) (cf. [49]).

Suppose $\delta \gamma$ is tangent to $\mathcal{M}_{2 n}$ at $\gamma$. By (36), $\mathrm{d} \Psi_{\gamma}(\delta \gamma)=\left[\partial+b+u, g^{-1} \delta g\right]$ is in $V_{n}$. So $C:=g^{-1} \delta g$ satisfies (37).

Suppose $C$ satisfies (37). Let $\eta_{i}$ denote the $i$-th column of $g C$. Please note that $\xi$ is tangent to $\mathcal{M}_{2 n}$ at $\gamma$ if and only if

$$
\left\{\begin{array}{l}
\omega\left(\xi_{x}^{(i)}, \gamma_{x}^{(j)}\right)+\omega\left(\gamma_{x}^{(i)}, \xi_{x}^{(j)}\right)=0, \quad 1 \leq i, j \leq n-1, \\
\omega\left(\xi_{x}^{(n-1)}, \gamma_{x}^{(n)}\right)+\omega\left(\gamma_{x}^{(n-1)}, \xi_{x}^{(n)}\right)=0 .
\end{array}\right.
$$

To prove $\eta_{1}$ satisfies (38), we let $\rho=\left[\partial_{x}+b+u, C\right]$. Then

$$
(g C)_{x}=g_{x} C+g C_{x}=g C(b+u)+g \rho .
$$

Since $\rho \in V_{n}, \eta_{i}=\left(\eta_{1}\right)_{x}^{(i-1)}$ for $1 \leq i \leq n+1$. By

$$
(g C)^{t} S_{n} g+g^{t} S_{n} g C=C^{t} g^{t} S_{n} g+g^{t} S_{n} g C=C^{t} S_{n}+S_{n} C=0,
$$

so $\eta_{1}$ satisfies (38).

By (14) and (33), we see that both $P_{2 j-1,0}(u)-\eta_{j}(u)$ and $Q_{2 j-1,0}(u)-\xi_{j}(u)$ satisfy (37). So it follows from Theorem 4 that

$$
\gamma_{t}=g\left(P_{2 j-1,0}(u)-\eta_{j}(u)\right) e_{1}, \quad \gamma_{t}=g\left(Q_{2 j-1,0}(u)-\xi_{j}(u)\right) e_{1}
$$

are flows on $\mathcal{M}_{2 n}$. Since $\eta_{j}(u)$ and $\xi_{j}(u)$ are all strictly upper triangular, we have

$$
\eta_{j}(u) e_{1}=\xi_{j}(u) e_{1}=\zeta_{j}(u) e_{1}=0 .
$$

Hence we have the following.

Proposition 10. Let $g$ and $u$ be the Lagrangian moving frame and Lagrangian curvature along $\gamma \in \mathcal{M}_{2 n}$ respectively. Then

$$
\begin{aligned}
& \gamma_{t}=g\left(P_{2 j-1,0}(u)-\eta_{j}(u)\right) e_{1}=g P_{2 j-1,0}(u) e_{1}, \\
& \gamma_{t}=g\left(Q_{2 j-1,0}(u)-\xi_{j}(u)\right) e_{1}=g Q_{2 j-1,0}(u) e_{1},
\end{aligned}
$$

are Lagrangian curve flows on $\mathcal{M}_{2 n}$, where $P_{2 j-1,0}(u), Q_{2 j-1,0}(u), \eta_{j}(u)$, and $\xi_{j}(u)$ are given by (12), (29), (21), and (33) respectively.

We call (39) and (40) the (2j-1)-th Lagrangian curve flow on $\mathcal{M}_{2 n}$ of C-type and Atype respectively.

\section{Example 7. Lagrangian curve flows of C-type}

(i) When $n=1, s p(2)=s l(2, \mathbb{R})$, the symplectic form $\omega(\xi, \eta)$ defined by (1) is $\operatorname{det}(\xi, \eta)$, $\gamma \in \mathcal{M}_{2}$ if and only if $\gamma$ satisfies $\operatorname{det}\left(\gamma, \gamma_{x}\right)=1$, and the Lagrangian parameter, moving frame, and curvature for $\gamma \in \mathcal{M}_{2}$ are the central affine parameter, moving frame and curvature respectively. The third $\hat{C}_{1}^{(1)}-K d V$ is the $K d V$,

$$
q_{t}=\frac{1}{4}\left(q_{x x x}-6 q q_{x}\right) .
$$

The third Lagrangian curve flow of C-type on $\mathcal{M}_{2}$ is

$$
\gamma_{t}=\frac{1}{4}\left(u_{1}\right)_{x} \gamma-\frac{1}{2} u_{1} \gamma_{x}
$$


which is the third central affine curve flow on the affine plane (cf. [47]). Moreover, if $\gamma$ is a solution of (42), then its Lagrangian curvature is a solution of the $K d V(41)$.

(ii) Let $g=\left(\gamma, \gamma_{x}, \gamma_{x x}, g_{4}\right)$ be the Lagrangian moving frame of $\gamma \in \mathcal{M}_{4}$, and $u_{1}, u_{2}$ the Lagrangian curvatures as in Example 2. From Example 5, we see that the first column of $P_{3,0}(u)$ is

$$
\left(-\frac{3}{8}\left(u_{1}\right)_{x}, \frac{1}{4} u_{1}, 0,1\right)^{t}
$$

So the third Lagrangian curve flow of C-type on $\mathcal{M}_{4}$ is

$$
\gamma_{t}=-\frac{3}{8}\left(u_{1}\right)_{x} \gamma+\frac{1}{4} u_{1} \gamma_{x}+g_{4}
$$

where $g_{4}$ is the fourth column of the Lagrangian frame of $\gamma$. This is the curve flow (3) for $n=2$ because $g_{4}=\gamma_{x x x}-u_{1} \gamma_{x}$ (given in Example 2).

Similar computation implies that the first column of $P_{5,0}(u)$ is

$$
\left(-\frac{5}{32}\left(u_{1}\right)_{x}^{(3)}+\frac{3}{8}\left(u_{2}\right)_{x}+\frac{5}{32} u_{1}\left(u_{1}\right)_{x}, \frac{1}{16}\left(u_{1}\right)_{x}^{(2)}-\frac{1}{4} u_{2}-\frac{3}{32} u_{1}^{2}, \frac{1}{8}\left(u_{1}\right)_{x},-\frac{1}{4} u_{1}\right)^{t} .
$$

Hence the fifth Lagrangian curve flow of C-type on $\mathcal{M}_{4}$ is

$$
\begin{aligned}
\gamma_{x}= & \left(-\frac{5}{32}\left(u_{1}\right)_{x}^{(3)}+\frac{3}{8}\left(u_{2}\right)_{x}+\frac{5}{32} u_{1}\left(u_{1}\right)_{x}\right) \gamma \\
& +\left(\frac{1}{16}\left(u_{1}\right)_{x}^{(2)}-\frac{1}{4} u_{2}-\frac{3}{32} u_{1}^{2}\right) \gamma_{x}+\frac{1}{8}\left(u_{1}\right)_{x} \gamma_{x x}-\frac{1}{4} u_{1} g_{4} .
\end{aligned}
$$

(iii) We use Equation (10) to compute $P_{1, i}(u)$ and the first column of $P_{3,0}(u)$ for general $n$. Then we see that the third Lagrangian curve flow of C-type on $\mathcal{M}_{2 n}$ for $n \geq 3$ is (3).

\section{Example 8. Lagrangian curve flows of A-type}

We use the algorithm given in Theorem 3 to compute $Q_{1, i}(u)$. Then we use these $Q_{1, i}(u)^{\prime}$ s to compute $Q_{i, 0}(u)$. Then we obtain the following:

(i) The third Lagrangian curve flow of A-type on $\mathcal{M}_{4}$ is

$$
\gamma_{t}=\gamma_{x}^{(3)}-u_{1} \gamma_{x}
$$

The fifth Lagrangian curve flow of A-type on $\mathcal{M}_{4}$ is

$$
\begin{aligned}
\gamma_{t}= & \left(-\frac{5}{3}\left(u_{2}\right)_{x}-\frac{1}{9}\left(u_{1}\right)_{x}^{(3)}+\frac{1}{6} u_{1}\left(u_{1}\right)_{x}\right) \gamma-\frac{1}{9}\left(6 u_{2}+\left(u_{1}\right)_{x}^{(2)}+u_{1}^{2}\right) \gamma_{x} \\
& +\frac{1}{3}\left(u_{1}\right)_{x} \gamma_{x x}-\frac{2}{3} u_{1} g_{4} .
\end{aligned}
$$

(ii) The third Lagrangian curve flow of A-type on $\mathcal{M}_{2 n}(n \geq 2)$ is (4). Since $\gamma_{x}^{(3)}=u_{1} \gamma_{x}+g_{4}$, (4) becomes (43) when $n=2$.

Theorem 4 (1) states that $g \xi$ is tangent to $\mathcal{M}_{2 n}$ at $\gamma$ if and only if there is a $C$ satisfying (37) and $\xi=C e_{1}$. So to get a better description of the tangent space of $\mathcal{M}_{2 n}$ at $\gamma$, we need to understand properties of $C$ that satisfies (37).

Theorem 5. Let $u \in C^{\infty}\left(\mathbb{R}, V_{n}\right)$ and $v=\sum_{i=1}^{n} v_{i} e_{n+i, n+1-i}: \mathbb{R} \rightarrow V_{n}^{t}$ a smooth map. Let $\pi_{0}$ : $s p(2 n) \rightarrow V_{n}^{t}$ be the linear projection onto $V_{n}^{t}$ defined by

$$
\pi_{0}(y)=\sum_{i=1}^{n} y_{n+i, n+1-i} e_{n+i, n+1-i}, \quad y=\left(y_{i j}\right) .
$$




$$
\begin{aligned}
\text { If } C=\left(C_{i j}\right): \mathbb{R} \rightarrow & s p(2 n) \text { satisfies } \\
& {\left[\partial_{x}+b+q, C\right] \in C^{\infty}\left(\mathbb{R}, V_{n}\right), \quad \pi_{0}(C)=v, }
\end{aligned}
$$

then we have the following:

(i) There exists differential polynomial $\phi_{i j}(u, v)$ that is linear in $v$ such that $C_{i j}=\phi_{i j}(u, v)$ for all $1 \leq i, j \leq 2 n$, and $\phi_{n+i, n+1-i}(u, v)=v_{i}$ for $1 \leq i \leq 2 n$.

(ii) $\phi_{2 i, 1}(u, v)=v_{i}+\phi_{i}$ for $1 \leq i \leq n$, where $\phi_{i}$ 's are differential polynomials in $u, v_{i+1}, \cdots, v_{n}$.

(iii) There exist differential polynomials $h_{2 i+1}$ for $0 \leq i \leq n-1$ such that

$$
C_{2 i+1,1}=h_{2 i+1}\left(u, C_{2 i+2,1}, \cdots, C_{2 n, 1}\right) .
$$

(iv) $C_{i, j}$ 's are differential polynomials of $u, C_{21}, \cdots, C_{2 n, 1}$.

Conversely, given $u \in C^{\infty}\left(\mathbb{R}, V_{n}\right)$ and $v \in C^{\infty}\left(\mathbb{R}, V_{n}^{t}\right)$, define $C=\left(C_{i j}\right)$ by $C_{i j}=\phi_{i j}(u, v)$ for $1 \leq i, j \leq 2 n$. Then $C$ satisfies (45).

Proof. Let $\mathcal{G}_{i}=\operatorname{span}\left\{e_{j, i+j} \mid 1 \leq i+j \leq 2 n\right\}$. For $\xi \in s p(2 n)$, we use $\xi_{\mathcal{G}_{i}}$ to denote the $\mathcal{G}_{i}$-component of $\xi$ with respect to $s p(2 n)=\oplus_{i=1-2 n}^{2 n-1} \mathcal{G}_{i}$, and write $C=\sum_{i=1-2 n}^{2 n-1} C_{i}, C_{i} \in \mathcal{G}_{i}$. Set $\left[\partial_{x}+b+u, C\right]=\sum_{i=1}^{n} \eta_{i} e_{n+1-i, n+i}$. Then

$$
\left(C_{i}\right)_{x}+\left[b, C_{i+1}\right]+[u, C]_{\mathcal{G}_{i}}=\left\{\begin{array}{l}
\eta_{j} e_{n+1-j, n+j,}, i=2 j-1, \\
0, \quad \text { else. }
\end{array}\right.
$$

We prove (i) by induction. When $i=1-2 n$, we have $C_{2 n, 1}=v_{n}$. From $\left(C_{1-2 n}\right)_{x}+$ $\left[b, C_{2-2 n}\right]=0$, we get $C_{2 n-1,1}=-C_{2 n, 2}=-\frac{1}{2}\left(v_{n}\right)_{x}$. For $j<0, a d(b): \mathcal{G}_{2 j} \rightarrow \mathcal{G}_{2 j-1}$ is a bijection, and $\operatorname{dim}\left(a d(b)\left(\mathcal{G}_{2 j+1}\right)\right)=\operatorname{dim}\left(\mathcal{G}_{2 j}\right)=\operatorname{dim}\left(\mathcal{G}_{2 j+1}\right)-1$. Then by (46) and induction, $C_{j}(j<0)$ are differential polynomials in $u, v_{i}$ and the linear system (46) implies (ii).

Please note that $a d(b): \mathcal{G}_{0} \rightarrow \mathcal{G}_{-1}$ is bijection, and $[u, C]_{\mathcal{G}_{-1}}$ depends only in $u, v_{1}, \cdots, v_{n}$. Hence $C_{0}$ can be solved uniquely from $C_{i}, i<0$. This proves (iii).

For $j>0, a d(b): \mathcal{G}_{2 j+1} \rightarrow \mathcal{G}_{2 j}$ is a bijection. Hence $\mathcal{G}_{2 j+1}$ is a differential polynomial in $\mathcal{G}_{2 j}$. In addition, $a d(b): \mathcal{G}_{2 j+2} \rightarrow \mathcal{G}_{2 i+1}$ is an injection and $\operatorname{dim}\left(a d(b)\left(\mathcal{G}_{2 j+2}\right)\right)=\operatorname{dim}\left(\mathcal{G}_{2 j+1}\right)-1$. Then by induction, $C_{j}(j>0)$ are differential polynomials in $u, v_{1}, \cdots, v_{n}$. This proves (i). Moreover, from the argument, we see that $\eta_{i}$ 's are differential polynomials in $u$ and $v_{1}, \cdots, v_{n}$.

Statement (iv) is a consequence of (i) and (ii).

The proof of (i) to (iv) implies that the converse is also true.

Corollary 1. Let $u$ and $g$ be the Lagrangian curvature and frame of $\gamma \in \mathcal{M}_{2 n}$. Then $T_{\gamma} \mathcal{M}_{2 n}$ is the set of all $g \xi$, where $\xi=\left(\xi_{1}, \ldots, \xi_{2 n}\right)^{t}$ satisfies $\xi_{2 i+1}=h_{2 i+1}\left(u, \xi_{2 i+2}, \cdots, \xi_{2 n}\right)$ and $h_{2 i+1}$ is given in Proposition 5. In particular, the tangent space of $\mathcal{M}_{2 n}$ at $\gamma \in \mathcal{M}_{2 n}$ is isomorphic to $C^{\infty}\left(\mathbb{R}, \mathbb{R}^{n}\right)$.

Proof. It follows from Theorem 4 (1) and Proposition 5 (iv).

Corollary 2. Given $C_{1}, C_{2}: \mathbb{R} \rightarrow s p(2 n)$ satisfying (37), then we have the following:

(1) If the first columns of $C_{1}$ and $C_{2}$ are the same, then $C_{1}=C_{2}$.

(2) If $\pi_{0}\left(C_{1}\right)=\pi_{0}\left(C_{2}\right)$, then $C_{1}=C_{2}$, where $\pi_{0}$ is the projection defined by (44).

Proof. (1) follows from Proposition 5 (iv), and (2) follows from Proposition 5 (i).

It follows from Proposition 5 (i) that we have the following:

Corollary 3. Given smooth $u: \mathbb{R} \rightarrow V_{n}$ and $v: \mathbb{R} \rightarrow V_{n}^{t}$, there exists a unique $C: \mathbb{R} \rightarrow \operatorname{sp}(2 n)$ satisfying (45) and entries of $C$ are polynomial differentials of $u, v$ and linear in $v$. 
The above Corollary leads us to define a natural linear differential operator $P_{u}$ defined below.

Definition 4. Given $u \in C^{\infty}\left(\mathbb{R}, V_{n}\right)$, let

$$
P_{u}: C^{\infty}\left(\mathbb{R}, V_{n}^{t}\right) \rightarrow C^{\infty}(\mathbb{R}, s p(2 n))
$$

be the linear differential operator defined by $P_{u}(v)=$ the unique $C \in C^{\infty}(\mathbb{R}, s p(2 n))$ satisfies (45).

It follows from the definition of $P_{u}$ and Theorem 5 that we have the following:

Proposition 11. Let $u \in C^{\infty}\left(\mathbb{R}, V_{n}\right)$. Then

(i) C satisfies (37) if and only if $C=P_{u}(v)$ for some $v \in C^{\infty}\left(\mathbb{R}, V_{n}^{t}\right)$.

(ii) If $C$ satisfies (37), then $C=P_{u}\left(\pi_{0}(C)\right)$.

Example 9. Let $u=u_{1} e_{23}+u_{2} e_{14} \in C^{\infty}\left(S^{1}, V_{2}\right)$, and $\xi=\xi_{1} e_{32}+\xi_{2} e_{41} \in C^{\infty}\left(S^{1}, V_{2}^{t}\right)$. We use the algorithm given in the proof of Proposition 5 to obtain:

$$
P_{u}(\xi)=\left(\begin{array}{cccc}
-\frac{1}{2}\left(\xi_{2}^{\prime \prime \prime}+3 \xi_{1}^{\prime}-u_{1} \xi_{2}^{\prime}\right) & C_{12} & C_{13} & C_{14} \\
\frac{1}{2} \xi_{2}^{\prime \prime}+\xi_{1} & -\frac{1}{2} \xi_{1}^{\prime} & C_{23} & -C_{13} \\
-\frac{1}{2} \xi_{2}^{\prime} & \xi_{1}^{\prime} & \frac{1}{2} \xi_{1}^{\prime} & C_{12} \\
\xi_{2} & \frac{1}{2} \xi_{2}^{\prime} & \frac{1}{2} \xi_{2}^{\prime \prime}+\xi_{1} & \frac{1}{2}\left(\xi_{2}^{\prime \prime \prime}+3 \xi_{1}^{\prime}-u_{1} \xi_{2}^{\prime}\right)
\end{array}\right)
$$

and

$$
\begin{aligned}
C_{12}= & -\frac{1}{2} \xi_{2}^{(4)}-\frac{3}{2} \xi_{1}^{\prime \prime}+\frac{1}{2}\left(u_{1} \xi_{2}^{\prime}\right)^{\prime}+u_{2} \xi_{2}, \\
C_{23}= & -\frac{1}{2} \xi_{2}^{(4)}-2 \xi_{1}^{\prime \prime}+\frac{1}{2}\left(u_{1} \xi_{2}^{\prime}\right)^{\prime}+u_{2} \xi_{2}+u_{1} \xi_{1}, \\
C_{13}= & -\frac{1}{2} \xi_{2}^{(5)}-\frac{3}{2} \xi_{1}^{(3)}+\frac{1}{2}\left(u_{1} \xi_{2}^{\prime}\right)^{\prime \prime}+\left(u_{2} \xi_{2}\right)^{\prime}+\frac{1}{2} u_{2} \xi_{2}^{\prime}, \\
C_{14}= & -\frac{1}{2} \xi_{2}^{(6)}-\frac{3}{2} \xi_{1}^{(4)}+\frac{1}{2}\left(u_{1} \xi_{2}^{\prime}\right)^{(3)}+\frac{1}{2} u_{1} \xi_{2}^{(4)}+\left(u_{2} \xi_{2}\right)^{\prime \prime}+u_{2} \xi_{2}^{\prime \prime}+\frac{1}{2} u_{2}^{\prime} \xi_{2}^{\prime} \\
& +u_{2} \xi_{1}+\frac{3}{2} u_{1} \xi_{1}^{\prime \prime}-\frac{1}{2} u_{1}\left(u_{1} \xi_{2}^{\prime}\right)^{\prime}-u_{1} u_{2} \xi_{2} .
\end{aligned}
$$

Example 10 (Tangent space of $\mathcal{M}_{4}$ at $\gamma$ ). Let $u=u_{1} e_{23}+u_{2} e_{14}$ and $g=\left(\gamma, \gamma^{\prime}, \gamma^{\prime \prime}, g_{4}\right)$ be the Lagrangian curvature and frame along $\gamma$ as in Example 2, where $g_{4}=\gamma^{\prime \prime \prime}-u_{1} \gamma^{\prime}$. It follows from Corollary 1 and Proposition 11 that the tangent space of $\mathcal{M}_{4}$ at $\gamma$ is

$$
\left\{g P_{u}(\xi) e_{1} \mid \xi \in C^{\infty}\left(\mathbb{R}, V_{n}^{t}\right)\right\}
$$

We use the formula of $P_{u}(\xi)$ given in Example 9. Set $\eta_{1}=\frac{1}{2} \xi_{2}^{\prime \prime}+\xi_{1}$ and $\eta_{2}=\xi_{2}$. Then we have $\xi_{1}=\eta_{1}-\frac{1}{2} \eta_{2}^{\prime \prime}$ and $\xi_{2}=\eta_{2}$. So the first column of $P_{u}(\xi)$ is

$$
\left(\frac{1}{4} \eta_{2}^{\prime \prime \prime}-\frac{3}{2} \eta_{1}^{\prime}+\frac{1}{2} u_{1} \eta_{2}^{\prime}, \eta_{1},-\frac{1}{2} \eta_{2}^{\prime}, \eta_{2}\right)^{t}
$$

Hence the tangent space of $\mathcal{M}_{4}$ at $\gamma$ is the space of

$$
\left(\frac{1}{4} \eta_{2}^{\prime \prime \prime}-\frac{3}{2} \eta_{1}^{\prime}+\frac{1}{2} u_{1} \eta_{2}^{\prime}\right) \gamma+\eta_{1} \gamma_{x}-\frac{1}{2} \eta_{2}^{\prime} \gamma_{x x}+\eta_{2} g_{4}
$$

where $\eta_{1}, \eta_{2}$ are smooth functions. 
Proposition 12. Let $u \in C^{\infty}\left(\mathbb{R}, V_{n}\right), P_{2 j-1,0}(u), Q_{2 j-1,0}(u), \xi_{j}(u), \eta_{j}(u)$ as in (12), (29), (21), (33) respectively. Then

$$
\begin{gathered}
P_{u}\left(\pi_{0}\left(P_{2 j-1,0}(u)\right)\right)=P_{2 j-1,0}(u)-\eta_{j}(u), \\
P_{u}\left(\pi_{0}\left(Q_{2 j-1,0}(u)\right)\right)=Q_{2 j-1,0}(u)-\xi_{j}(u),
\end{gathered}
$$

and the $(2 j-1)-\operatorname{th} \hat{C}_{n}^{(1)}-K d V$ and $\hat{A}_{2 n-1}^{(2)}-K d V$ flows can be written respectively as

$$
\begin{aligned}
& u_{t}=\left[\partial_{x}+b+u, P_{u}\left(\pi_{0}\left(P_{2 j-1,0}(u)\right)\right)\right], \\
& u_{t}=\left[\partial_{x}+b+u, P_{u}\left(\pi_{0}\left(Q_{2 j-1,0}(u)\right)\right)\right] .
\end{aligned}
$$

Proof. It follows from (21) and (34) that both $P_{2 j-1,0}(u)-\eta_{j}(u)$ and $Q_{2 j-1,0}(u)-\xi_{j}(u)$ satisfies $\left[\partial_{x}+b+q, C\right]$ is $V_{n}$-valued. Proposition follows from Proposition 11 (ii).

\section{Theorem 6.}

(i) If $\gamma \in \mathcal{M}_{2 n}$ is a solution of the (2j-1)-th Lagrangian curve flow (39) of C-type ((40) of A-type resp.), then its Lagrangian curvature $u$ is a solution of the $(2 j-1)-t h \hat{C}_{n}^{(1)}-K d V$ flow (22) $\left(\hat{A}_{2 n-1}^{(2)}-K d V\right.$ flow (34) resp.).

(ii) Let $u \in C^{\infty}\left(\mathbb{R}^{2}, V_{n}\right)$ be a solution of (22) ((34) resp.), and $g: \mathbb{R}^{2} \rightarrow S p(2 n)$ a solution of (23) ((35) resp.). Then $\gamma(x, t):=g(x, t) e_{1}$ is solution of the $(2 j-1)$-th isotropic curve flow of C-type (39) (A-type (40) resp.) with Lagrangian curvature $u(\cdot, t)$ and Lagrangian moving frame $g(\cdot, t)$.

(iii) Let $q$ be a solution of the $(2 j-1)$-th $\hat{C}_{n}^{(1)}$-flow (14) $\left(\hat{A}_{2 n-1}^{(2)}\right.$-flow (31) resp.), and $g: \mathbb{R}^{2} \rightarrow$ $S p(2 n)$ a solution of (15) ((32) resp.). Then $\gamma(x, t)=g(x, t) e_{1}$ is a solution of the $(2 j-1)$-th Lagrangian curve flow (39) of C-type ((4) of A-type resp).

Proof. We claim that $Z:=g^{-1} g_{t}$ satisfies (37). Since $g^{-1} g_{x}=b+u$ with $u \in V_{n}$, a direct computation implies that

$$
y:=\left(g^{-1} g_{x}\right)_{t}=-Z(b+u)-g^{-1} g_{x t}
$$

is $V_{n}$-valued. By (47), we obtain

$$
Z_{x}+[b+u, Z]=-g^{-1} g_{x} g^{-1} g_{t}+g^{-1} g_{x t}=y,
$$

which is $V_{n}$-valued. So $Z$ satisfies (37). By definition of $\eta_{j}(u), P_{2 j-1}(u)-\eta_{j}(u)$ also satisfies (37). The first column of $g Z e_{1}$ is $\gamma_{t}$, which is $P_{2 j-1}(u) e_{1}$. Since $\eta_{j}(u)$ is strictly upper triangular, the first column of $P_{2 j-1}(u)-\eta_{j}(u)$ is also $P_{2 j-1}(u) e_{1}$. It follows from Corollary 2 that $Z=P_{2 j-1}(u)-\eta_{j}(u)$. Hence we have proved $g$ satisfies (23). By Proposition $6, u$ is a solution of (22). This proves (i).

Since $g^{-1} g_{x}=b+u, g(\cdot, t)$ is the Lagrangian frame along $\gamma=g e_{1}$. So $\gamma_{t}=g_{t} e_{1}=$ $g\left(P_{2 j-1}(u)-\eta_{j}(u)\right) e_{1}=g P_{2 j-1}(u) e_{1}$. This proves (ii). (iii) is proved similarly.

Remark 1. We use the same proof as in [49] for the n-dimensional central affine curve flow to show that solutions of the Cauchy problem of (22) give solutions of the Cauchy problem for Lagrangian curve flow (39) with both rapidly decaying and periodic initial data. Similar results hold for the Lagrangian curve flows (34) and (40).

\section{Darboux Transforms for the $\hat{C}_{n}^{(1)}$-Hierarchy}

In this section, we use the loop group factorization method given in [54] to construct Darboux transformations for the $\hat{C}_{n}^{(1)}{ }_{-}, \hat{C}_{n}^{(1)}-\mathrm{KdV}$, and the Lagrangian curve flows of $\mathrm{C}$ type. We also give a Permutability formula for these Darboux transforms. To use this 
method, we need to identify the loop groups, find simple rational elements, and write down formulas for the factorizations.

Let $\left(\hat{\mathbb{C}}_{n}^{(1)}\right)$ + denote the group of holomorphic maps $f: \mathbb{C} \rightarrow G L(2 n, \mathbb{C})$ satisfying the $S p(2 n)$-reality condition (7), i.e.,

$$
\overline{f(\bar{\lambda})}=f(\lambda), \quad f(\lambda)^{t} S_{n} f(\lambda)=S_{n},
$$

and $\mathbb{R} \hat{\mathbb{C}}_{n}^{(1)}$ the group of rational maps $f: \mathbb{C} \rightarrow G L(2 n, \mathbb{C})$ satisfying $(7)$ and $f(\infty)=\mathrm{I}$. Then the Lie algebras of $\left(\hat{\mathbb{C}}_{n}^{(1)}\right)+$ and $\mathbb{R} \hat{\mathbb{C}}_{n}^{(1)}$ are contained in $\left(\hat{C}_{n}^{(1)}\right)+$ and $\left(\hat{C}_{n}^{(1)}\right)$ - respectively.

Next Proposition gives the uniqueness of factorization.

Proposition 13. Let $f_{1}, g_{1} \in\left(\hat{\mathbb{C}}_{n}^{(1)}\right)_{+}$, and $f_{2}, g_{2} \in \mathbb{R} \hat{\mathbb{C}}_{n}^{(1)}$. If $f_{1} f_{2}=g_{1} g_{2}$, then $f_{1}=g_{1}$ and $f_{2}=g_{2}$.

Proof. Let $h:=g_{1}^{-1} f_{1}=g_{2} f_{2}^{-1}$. Then $h$ is both holomorphic for $\lambda \in \mathbb{C}$ and at $\lambda=\infty$. So $h$ is constant. However, at $\lambda=\infty, h=\mathrm{I}$. Therefore, $h \equiv \mathrm{I}$.

The following result was proved in [54] for soliton hierarchies constructed from a splitting of loop algebras. So it works for both the $\hat{C}_{n}^{(1)}$ - and $\hat{A}_{2 n-1}^{(2)}$-hierarchies given in Section 3.

Theorem 7 ([54]). Let $F(x, t, \lambda)$ be a frame of a solution $q$ of (14) ((31) resp.) and $g \in \mathbb{R} \hat{\mathbb{C}}_{n}^{(1)}$. Then there exists an open neighborhood $\mathcal{O}$ of $(0,0)$ such that we can factor

$$
g(\lambda) F(x, t, \lambda)=\tilde{F}(x, t, \lambda) \tilde{g}(x, t, \lambda)
$$

with $\tilde{F}(x, t, \cdot)$ in $\left(\hat{C}_{n}^{(1)}\right)+$ and $\tilde{g}(x, t, \cdot) \in \mathbb{R} \hat{\mathbb{C}}_{n}^{(1)}$ for all $(x, t) \in \mathcal{O}$. Moreover, write

$$
\tilde{g}(x, t, \lambda)=\mathrm{I}+g_{-1}(x, t) \lambda^{-1}+g_{-2}(x, t) \lambda^{-2}+\cdots .
$$

Then $\tilde{q}=q+\left[g_{-1}, \beta\right]$ is a new solution of (14) ((31) resp.) and $\tilde{F}$ is a frame of $\tilde{q}$, where $\beta=e_{1,2 n}\left(\beta=\frac{1}{2}\left(e_{1,2 n-1}+e_{2,2 n}\right)\right.$ resp. $)$.

Theorem 8. Let $g \bullet q$ denote the solution of (14) ((31) resp.) constructed from the frame $F(x, t, \lambda)$ of solution $q$ of $(14)\left((31)\right.$ resp.) satisfying $F(0,0, \lambda)=I$. Then $g \bullet q$ defines an action of $\mathbb{R} \hat{\mathbb{C}}_{n}^{(1)}$ on the space of solutions of (14) ((31) resp.).

Proof. It suffices to prove that $(g f) \bullet q=g \bullet(f \bullet q)$ for $f, g \in \mathbb{R} \hat{\mathbb{C}}_{n}^{(1)}$. Assume that

$$
\begin{aligned}
f(\lambda) F(x, t, \lambda) & =F_{1}(x, t, \lambda) \tilde{f}(x, t, \lambda), \\
g(\lambda) F_{1}(x, t, \lambda) & =\tilde{F}(x, t, \lambda) \tilde{g}(x, t, \lambda),
\end{aligned}
$$

where $\tilde{f}(x, t, \cdot), \tilde{g}(x, t, \cdot)$ are in $\mathbb{R} \hat{\mathbb{C}}_{n}^{(1)}$ and $F_{1}(x, t, \lambda) \tilde{F}(x, t, \lambda)$ are holomorphic for $\lambda \in \mathbb{C}$. It follows from Theorem 7 that we have

$$
\begin{aligned}
f \bullet q & =q+\left[\tilde{f}_{-1}, \beta\right], \\
g \bullet(f \bullet q) & =f \bullet q+\left[\tilde{g}_{-1}, \beta\right]=q+\left[\tilde{f}_{-1}+\tilde{g}_{-1}, \beta\right]
\end{aligned}
$$

are solutions of (14), where

$$
\begin{aligned}
& \tilde{f}(x, t, \lambda)=\mathrm{I}+\tilde{f}_{-1}(x, t) \lambda^{-1}+\tilde{f}_{-2}(x, t) \lambda^{-2}+\cdots, \\
& \tilde{g}(x, t, \lambda)=\mathrm{I}+\tilde{g}_{-1}(x, t) \lambda^{-1}+\tilde{g}_{-2}(x, t) \lambda^{-2}+\cdots .
\end{aligned}
$$


To obtain $(g f) \bullet q$, we factor

$$
(g f) F=g(f F)=g\left(F_{1} \tilde{f}\right)=\left(g F_{1}\right) \tilde{f}=(\tilde{F} \tilde{g}) \tilde{f}=\tilde{F}(\tilde{g} \tilde{f}) .
$$

Since $\tilde{f}(x, t, \cdot)$ and $\tilde{g}(x, t, \cdot)$ are in $\mathbb{R} \hat{\mathbb{C}}_{n}^{(1)}, \tilde{g} \tilde{f}$ is in $\mathbb{R} \hat{\mathbb{C}}_{n}^{(1)}$. Please note that the coefficient of $\lambda^{-1}$ of $\tilde{g} \tilde{f}$ is $\tilde{f}_{-1}+\tilde{g}_{-1}$. Hence it follows from Theorem 7 that we have

$$
(g f) \bullet q=q+\left[\beta, \tilde{f}_{-1}+\tilde{g}_{-1}\right] .
$$

So we have proved that $(g f) \bullet q=g \bullet(f \bullet q)$.

Given a linear subspace $V$ of $\mathbb{R}^{2 n}$, let

$$
V^{\perp}=\left\{y \in \mathbb{R}^{2 n} \mid \omega(\xi, y)=0 \quad \text { for all } \xi \in V\right\} .
$$

Lemma 1. Let $\mathbb{R}^{2 n}=V_{1} \oplus V_{2}$ be a direct sum of linear subspaces, and $\pi$ the projection of $\mathbb{R}^{2 n}$ onto $V_{1}$ along $V_{2}$. Then we have $\omega(\pi X, Y)=\omega\left(X, \pi_{S} Y\right)$, where

$$
\pi_{s}=S_{n}^{-1} \pi^{t} S_{n}
$$

is a projection of $\mathbb{R}^{2 n}$ onto $V_{2}^{\perp}$ regarding $\mathbb{R}^{2 n}=V_{2}^{\perp} \oplus V_{1}^{\perp}$.

Proof. Please note that

$$
\omega(\pi(X), Y)=X^{t} \pi^{t} S_{n} Y=\omega\left(X, S_{n}^{-1} \pi^{t} S_{n}(Y)\right)=\omega\left(X, \pi_{s} Y\right),
$$

where $\omega$ is the symplectic form defined by (1).

If $\omega\left(\pi_{s} X, Y\right)=0$ for all $X \in \mathbb{R}^{2 n}$, then $\omega(X, \pi Y)=0$. Hence $\left(\operatorname{Im}\left(\pi_{s}\right)\right)^{\perp} \subset \operatorname{Ker}(\pi)=V_{2}$, which implies $\operatorname{Im}\left(\pi_{s}\right)=V_{2}^{\perp}$.

On the other hand, if $Y \in \operatorname{Ker}\left(\pi_{s}\right)$, then $\omega(\pi X, Y)=\omega\left(X, \pi_{S} Y\right)=0$ for any $X \in \mathbb{R}^{2 n}$. So $Y \in V_{1}^{\perp}$, which implies $\operatorname{Ker}\left(\pi_{s}\right)=V_{1}^{\perp}$.

We use Lemma 1 and a direct computation to get:

\section{Lemma 2.}

(1) A linear subspace of $\mathbb{R}^{2 n}$ is Lagrangian if and only if $V^{\perp}=V$.

(2) Let $\pi$ be a projection of $\mathbb{R}^{2 n}$. Then

$\operatorname{Im} \pi$ and $\operatorname{Ker} \pi$ are Lagrangian subspaces,

if and only if

$$
\pi_{s}=\mathrm{I}_{2 n}-\pi
$$

Given $\alpha \in \mathbb{R} \backslash 0$ and a projection $\pi$ of $\mathbb{R}^{2 n}$, let

$$
k_{\alpha, \pi}(\lambda)=\mathrm{I}+\frac{2 \alpha}{\lambda-\alpha}(\mathrm{I}-\pi)
$$

A direct computation implies that

$$
k_{\alpha, \pi}^{-1}(\lambda)=\mathrm{I}-\frac{2 \alpha}{\lambda+\alpha}(\mathrm{I}-\pi)
$$

Lemma 3. Given $\alpha \in \mathbb{R} \backslash 0$, if $\pi$ is a projection of $\mathbb{R}^{2 n}$ satisfying (48) then

$$
k_{\alpha, \pi}^{t}(\lambda) S_{n} k_{\alpha, \pi}(\lambda)=\frac{\lambda+\alpha}{\lambda-\alpha} S_{n} .
$$


Proof. Lemmas 1 and 2 (2) implies that $S_{n}^{-1} \pi^{t} S_{n}=\mathrm{I}-\pi$. So we have I $-\pi^{t}=S_{n} \pi S_{n}^{-1}$. Then a direct computation gives (52).

Lemma 4. Let $\alpha \in \mathbb{R} \backslash 0, \pi$ a projection satisfying (48), and $f: \mathbb{C} \rightarrow G L(2 n, \mathbb{C})$ a meromorphic map, holomorphic at $\lambda=\alpha,-\alpha$, and $f$ satisfying the $S p(2 n)$-reality condition (7). Let $\tilde{V}_{1}=$ $f(\alpha)^{-1}(\operatorname{Im} \pi)$, and $\tilde{V}_{2}=f(-\alpha)^{-1}(\operatorname{Ker} \pi)$. Assume that $\tilde{V}_{1} \cap \tilde{V}_{2}=\{0\}$. Let $\tilde{\pi}$ be the projection onto $\tilde{V}_{1}$ along $\tilde{V}_{2}$. Then

(1) $\tilde{V}_{1}$ and $\tilde{V}_{2}$ are Lagrangian subspaces,

(2) $\tilde{f}:=k_{\alpha, \pi} f k_{\alpha, \tilde{\pi}}^{-1}$ is holomorphic at $\lambda=\alpha,-\alpha$ and satisfies the $S p(2 n)$-reality condition (7).

Proof. Since $f$ satisfies the $S p(2 n)$-reality condition, $f(r) \in S p(2 n)$ for all $r \in \mathbb{R}$. Hence $f(r)^{-1}\left(V_{i}\right)$ is again a Lagrangian subspace. This proves (i).

By (51), we have

$$
\tilde{f}(\lambda)=\left(\mathrm{I}+\frac{2 \alpha}{\lambda-\alpha}(\mathrm{I}-\pi)\right) f(\lambda)\left(\mathrm{I}-\frac{2 \alpha}{\lambda+\alpha}(\mathrm{I}-\tilde{\pi})\right) .
$$

Please note that $\tilde{f}$ has a simple pole at $\lambda=\alpha$ and the residue of $\tilde{f}$ at $\lambda=\alpha$ is $2 \alpha(\mathrm{I}-\pi) f(\alpha) \tilde{\pi}$, which is zero because

$$
(\mathrm{I}-\pi) f(\alpha) \operatorname{Im} \tilde{\pi}=(\mathrm{I}-\pi) f(\alpha) f(\alpha)^{-1}(\operatorname{Im} \pi)=(\mathrm{I}-\pi)(\operatorname{Im} \pi)=0 .
$$

Similarly, $\tilde{f}$ has a simple pole at $\lambda=-\alpha$ and its residue is $2 \alpha \pi f(-\alpha)(\mathrm{I}-\tilde{\pi})$, which is again zero because its image is

$$
\pi f(-\alpha) \operatorname{Ker} \tilde{\pi}=\pi f(-\alpha) f(-\alpha)^{-1}(\operatorname{Ker} \pi)=0 .
$$

This proves $\tilde{f}$ is holomorphic at $\lambda=\alpha,-\alpha$.

It follows from (52) that $k_{\alpha, \pi}^{-1}=\frac{\lambda-\alpha}{\lambda+\alpha}\left(k_{\alpha, \pi}\right)_{s}$. Since $f$ satisfies $f(\lambda)^{-1}=(f(\lambda))_{s}$, a direct computation shows that $\tilde{f}^{-1}=\tilde{f}_{s}$. Hence $\tilde{f}$ satisfies (7).

Theorem 9 (Darboux transform for the $\hat{C}_{n}^{(1)}$-flow (14)).

Let $c \in S p(2 n)$ be a constant, $F(x, t, \lambda)$ the frame of a solution $q$ of (14) satisfying $F(0,0, \lambda)=$ $c, \alpha \in \mathbb{R} \backslash\{0\}$, and $\pi$ a projection of $\mathbb{R}^{2 n}$ satisfying (48). Let

$$
\tilde{V}_{1}(x, t)=F^{-1}(x, t, \alpha)(\operatorname{Im} \pi), \quad \tilde{V}_{2}(x, t)=F^{-1}(x, t,-\alpha)(\operatorname{Ker} \pi) .
$$

Then

(1) $\tilde{V}_{i}(x, t)$ are Lagrangian subspaces for all $(x, t) \in \mathbb{R}^{2}$,

(2) there is an open subset $\mathcal{O}$ of $(0,0)$ in $\mathbb{R}^{2}$ such that $\mathbb{R}^{2 n}=\tilde{V}_{1}(x, t) \oplus \tilde{V}_{2}(x, t)$ for all $(x, t) \in \mathcal{O}$,

(3) let $\tilde{\pi}(x, t)$ be the projection of $\mathbb{R}^{2 n}$ onto $\tilde{V}_{1}(x, t)$ along $\tilde{V}_{2}(x, t)$, and

$$
\tilde{F}(x, t, \lambda)=k_{\alpha, \pi} F(x, t, \lambda) k_{\alpha, \tilde{\pi}(x, t)}^{-1}(\lambda),
$$

then

$$
\tilde{q}=q+2 \alpha\left[e_{1,2 n}, \tilde{\pi}\right]
$$

is a new solution of (14) and $\tilde{F}$ is a frame for $\tilde{q}$.

In particular, we have

$$
k_{\alpha, \pi} \bullet q=q+2 \alpha\left[e_{1,2 n}, \tilde{\pi}\right]
$$

if $F$ is chosen so that $F(0,0, \lambda)=\mathrm{I}$.

Proof. Let $V_{1}=\operatorname{Im} \pi$, and $V_{2}=\operatorname{Ker} \pi$. By assumption, $V_{1}, V_{2}$ are Lagrangian. Since $F(x, t, \lambda)$ satisfies the $\operatorname{Sp}(2 n)$ reality condition (7) and $\alpha,-\alpha \in \mathbb{R},(1)$ follows. 
By assumption, $V_{1} \cap V_{2}=\{0\}$. Please note that $\tilde{V}_{1}(0,0)=F(0,0, \alpha)^{-1}\left(V_{1}\right)=c^{-1}\left(V_{1}\right)$ and $\tilde{V}_{2}(0,0)=c^{-1} V_{2}$. So $\left(c^{-1} V_{1}\right) \cap\left(c^{-1} V_{2}\right)=\{0\}$. Since $\mathcal{O}=\left\{(x, t) \mid \tilde{V}_{1}(x, t) \cap \tilde{V}_{2}(x, t)=\right.$ $\{0\}\}$ is an open subset of $\mathbb{R}^{2}$ and $(0,0) \in \mathcal{O}$, (2) follows.

(3) follows from Lemma 4 and Theorem 7.

Theorem 9 can be reformulated as follows:

Theorem 10. Let $q$ be a solution of (14), $\lambda \in \mathbb{R} \backslash 0$, and $D_{q, \lambda}$ the following linear system

$$
D_{q, \lambda}\left\{\begin{array}{l}
y_{x}=-\left(e_{1,2 n} \lambda+b+q\right) y \\
y_{t}=-\left(P^{2 j-1}(q, \lambda)\right)+y
\end{array}\right.
$$

Then (56) is solvable. Moreover, let $\left\{v_{1}, \ldots, v_{2 n}\right\}$ be a basis of $\mathbb{R}^{2 n}$ such that the span of $v_{1}, \ldots, v_{n}$ and the span of $v_{n+1}, \ldots, v_{2 n}$ are Lagrangian subspaces. Let $y_{i}$ be the solution of $D_{q, \alpha}$ with initial data $y_{i}(0,0)=v_{i}$, and $y_{n+i}$ the solution of $D_{q,-\alpha}$ with $y_{n+i}(0,0)=v_{n+i}$ for $1 \leq i \leq n$. Let $\tilde{V}_{1}(x, t)$ be the span of $y_{1}(x, t), \ldots, y_{n}(x, t)$, and $\tilde{V}_{2}(x, t)$ the span of $y_{n+1}(x, t), \ldots, y_{2 n}(x, t)$. Then

(1) $\tilde{V}_{i}(x, t)$ is Lagrangian for all $(x, t) \in \mathbb{R}^{2}$ and $i=1,2$,

(2) there is an open subset $\mathcal{O}$ of $(0,0)$ such that $\tilde{V}_{1}(x, t) \cap \tilde{V}_{2}(x, t)=0$ for all $(x, t) \in \mathcal{O}$,

(3) $\tilde{q}$ defined by (54) is a solution of (14) defined on $\mathcal{O}$, where $\tilde{\pi}$ is the projection onto $\tilde{V}_{1}(x, t)$ along $\tilde{V}_{2}(x, t)$.

\section{Remark 2 (Bäcklund transformations for the $\hat{C}_{n}^{(1)}$-flow).}

Let $q, \tilde{q}, F, \tilde{F}$ be as in Theorem 9. Then we have

$$
\left\{\begin{array} { l } 
{ F ^ { - 1 } F _ { x } = J + q , } \\
{ F ^ { - 1 } F _ { t } = B ( q , \lambda ) , }
\end{array} \quad \left\{\begin{array}{l}
\tilde{F}^{-1} \tilde{F}_{x}=J+\tilde{q}, \\
\tilde{F}^{-1} \tilde{F}_{t}=B(\tilde{q}, \lambda),
\end{array}\right.\right.
$$

where $B(q, \lambda)=\left(P^{2 j-1}(q, \lambda)\right)_{+}$. Let $\tilde{k}(x, t)=k_{\alpha, \tilde{\pi}(x, t)}$. Then it follows from (57) and (53) that we have

$$
\left\{\begin{array}{l}
\tilde{k}(J+q)-\tilde{k}_{x}=(J+\tilde{q}) \tilde{k}, \\
\tilde{k} B(q, \lambda)-\tilde{k}_{t}=B(\tilde{q}, \lambda) \tilde{k} .
\end{array}\right.
$$

Equate the residues of (58) at $\lambda=\alpha$ to get

$$
(B T)_{q, \lambda}\left\{\begin{array}{l}
\tilde{\pi}_{x}=\left[\alpha e_{1,2 n}-b-q, \tilde{\pi}\right]-2 \alpha\left[e_{1,2 n}, \tilde{\pi}\right] \tilde{\pi}, \\
\tilde{\pi}_{t}=B\left(q+2 \alpha\left[e_{1,2 n}, \tilde{\pi}\right], \alpha\right)(I-\tilde{\pi})-(I-\tilde{\pi}) B(q, \alpha),
\end{array}\right.
$$

which is the Bäcklund transformation for the $\hat{C}_{n}^{(1)}$-flow. Moreover,

(1) $(B T)_{q, \lambda}$ is solvable for $\tilde{\pi}$ if and only if $q$ is a solution of the $\hat{C}_{n}^{(1)}$-flow,

(2) if $\pi_{0}$ is a projection satisfies (49), then the solution $\tilde{\pi}$ of $(B T)_{q, \alpha}$ with initial data $\tilde{\pi}(0,0)=\pi_{0}$ also satisfies (49) and $\tilde{q}$ defined by (54) is also a solution of the $\hat{C}_{n}^{(1)}$-flow. In fact, $\tilde{q}=k_{\alpha, \pi} \bullet q$.

The following DTs for (22) is a consequence of Proposition 9 and Theorem 9.

\section{Theorem 11 (DT for $\left.\hat{C}_{n}^{(1)}-\mathrm{KdV}\right)$.}

Let $E$ be a frame of a solution $u$ of (22), $k_{\alpha, \pi}$ as in Lemma $3, \triangle: \mathbb{R}^{2} \rightarrow N_{n}^{+}$satisfying $\triangle_{t} \triangle^{-1}=\eta_{j}(u)$, and $q=\triangle^{-1} * u$. Let

$$
\tilde{V}_{i}(x, t)=\triangle^{-1}(x, t) E^{-1}\left(x, t, \alpha_{i}\right)\left(V_{i}\right)
$$


for $i=1,2$, and $\tilde{\pi}(x, t)$ the projection onto $\tilde{V}_{1}(x, t)$ along $\tilde{V}_{2}(x, t)$. Let $\tilde{q}$ be defined by (54), $\tilde{\triangle}: \mathbb{R}^{2} \rightarrow N_{n}^{+}$the unique map such that $\tilde{u}=\tilde{\triangle} * \tilde{q}$ is $V_{n}$ valued. Then $\tilde{u}$ is a solution of (22) and

$$
\tilde{E}(x, t, \lambda)=k_{\alpha, \pi}(\lambda) E(x, t, \lambda) \triangle(x, t) \tilde{k}_{\alpha, \tilde{\pi}(x, t)}^{-1}(\lambda) \tilde{\triangle}^{-1}(x, t)
$$

is a frame of $\tilde{u}$, where $*$ is defined by (18).

As a consequence of Theorems 9 and 6 (iii), we have

Theorem 12 (DT for Lagrangian curve flow of C-type).

Let $\gamma$ be a solution of the Lagrangian curve flow (39), and $g(\cdot, t)$ and $u(\cdot, t)$ the Lagrangian frame and curvature along $\gamma(\cdot, t)$. Let $E$ be the frame of the solution $u$ of $(22)$ satisfying $E(0,0, \lambda)=$ $g(0,0)$. Let $\triangle, k_{\alpha, \pi}$, and $\tilde{\pi}$ be as in Theorem 11. Then

$$
\tilde{\gamma}=(2 \pi-\mathrm{I}) g \triangle(2 \tilde{\pi}-\mathrm{I}) e_{1}
$$

is a new solution of (39).

\section{Example 11. [1-soliton solutions of C-type]}

First, we apply Theorem 9 to the trivial solution $q=0$ of the third $\hat{C}_{2}^{(1)}$-flow to construct 1-soliton solutions and their corresponding frames. Then we use Theorem 11 to construct solutions of the third $\hat{C}_{2}^{(1)}-K d V$ flow (5). In the end, we apply Theorem 12 to get explicit solutions of the third Lagrangian curve flow of C-type on $\mathbb{R}^{4}$ :

$$
\gamma_{t}=-\frac{3}{8}\left(u_{1}\right)_{x} \gamma+\frac{1}{4} u_{1} \gamma_{x}+g_{4}=-\frac{3}{8}\left(u_{1}\right)_{x} \gamma-\frac{3}{4} u_{x} \gamma_{x}+\gamma_{x x x}
$$

Please note that

$$
F(x, t, \lambda)=\exp \left(J x+J^{3} t\right)
$$

is a frame of the solution $q=0$ of the third $\hat{C}_{2}^{(1)}$-flow. We use $\lambda=z^{4}$ to write down $F(x, t, \lambda)$ in terms of known functions,

$$
F\left(x, t, z^{4}\right)=\frac{1}{4}\left(\begin{array}{cccc}
m_{1}(x, t, z) & z m_{2}(x, t, z) & z^{2} m_{3}(x, t, z), & z^{3} m_{4}(x, t, z) \\
\frac{1}{z} m_{4}(x, t, z) & m_{1}(x, t, z) & z m_{2}(x, t, z) & z^{2} m_{3}(x, t, z) \\
\frac{1}{z^{2}} m_{3}(x, t, z) & \frac{1}{z} m_{4}(x, t, z) & m_{1}(x, t, z) & z m_{2}(x, t, z) \\
\frac{1}{z^{3}} m_{2}(x, t, z) & \frac{1}{z^{2}} m_{3}(x, t, z) & \frac{1}{z} m_{4}(x, t, z) & m_{1}(x, t, z)
\end{array}\right)
$$

where

$$
\left(\begin{array}{l}
m_{1}(x, t, z) \\
m_{2}(x, t, z) \\
m_{3}(x, t, z) \\
m_{4}(x, t, z)
\end{array}\right)=\left(\begin{array}{cccc}
1 & 1 & 1 & 1 \\
1 & i & -1 & -i \\
1 & -1 & 1 & -1 \\
1 & -i & -1 & i
\end{array}\right)\left(\begin{array}{c}
e^{z x+z^{3} t} \\
e^{i\left(z x-z^{3} t\right)} \\
e^{-\left(z x+z^{5} t\right)} \\
e^{-i\left(z x-z^{3} t\right)}
\end{array}\right)
$$

(Although the entries of $F\left(x, t, z^{3}\right)$ involves $z^{i}$ in the denominators, use power series expansion and a simple computation to see that they are holomorphic at $z=0$ ).

Next we apply DTs for the third $\hat{C}_{2}^{(1)}$-flow to the trivial solution $q=0$ and $z=1$. Let $\pi$ be the projection onto $V_{1}$ along $V_{2}$, where

$$
V_{1}=\left\{\left(\begin{array}{l}
1 \\
0 \\
1 \\
0
\end{array}\right),\left(\begin{array}{c}
1 \\
0 \\
-1 \\
0
\end{array}\right)\right\}, \quad V_{2}=\left\{\left(\begin{array}{l}
0 \\
1 \\
0 \\
1
\end{array}\right),\left(\begin{array}{c}
0 \\
1 \\
0 \\
-1
\end{array}\right)\right\}
$$


Then $\tilde{\pi}$ is the projection onto $\tilde{V}_{1}$ along $\tilde{V}_{2}$, where

$$
\begin{aligned}
\tilde{V}_{1} & =E(x, t, 1)^{-1} V_{1}=\operatorname{span}\left\{p_{1}, p_{2}\right\} \\
& =\operatorname{span}\left\{\left(\begin{array}{c}
\cosh (x+t) \\
-\sinh (x+t) \\
\cosh (x+t) \\
-\sinh (x+t)
\end{array}\right),\left(\begin{array}{c}
\cos (x-t) \\
-\sin (x-t) \\
-\cos (x-t) \\
\sin (x-t)
\end{array}\right)\right\}, \\
\tilde{V}_{2} & =E(x, t, 1)^{-1} V_{1}=\operatorname{span}\left\{p_{3}, p_{4}\right\} \\
& =\operatorname{span}\left\{\left(\begin{array}{c}
\sinh (x+t) \\
\cosh (x+t) \\
\sinh (x+t) \\
\cosh (x+t)
\end{array}\right),\left(\begin{array}{c}
-\sin (x-t) \\
\cos (x-t) \\
\sin (x-t) \\
-\cos (x-t)
\end{array}\right)\right\} .
\end{aligned}
$$

From a direct computation, we have

$$
\begin{aligned}
\tilde{\pi}(x, t) & =\left(\tilde{p}_{1}, \tilde{p}_{2}, 0,0\right)\left(\tilde{p}_{1}, \tilde{p}_{2}, \tilde{p}_{3}, \tilde{p}_{4}\right)^{-1} \\
& =\frac{1}{2}\left(\frac{1}{\sqrt{y_{1}}} \tilde{p}_{1},\left(1+\frac{y_{2}}{y_{3}}\right) \tilde{p}_{2}, 0, \frac{y_{2}}{y_{3}} \tilde{p}_{2}\right)\left(\begin{array}{c}
\frac{1}{\sqrt{y_{1}}} \tilde{p}_{1}^{t} \\
\tilde{p}_{2}^{t} \\
0 \\
\tilde{p}_{4}^{t}
\end{array}\right),
\end{aligned}
$$

where

$$
\begin{aligned}
& y_{1}=\cosh (2(x+t)) \\
& y_{2}=\sin (2(x-t)) \\
& y_{3}=(1-\sin (2(x-t)))\left(1+\frac{1}{2} \sin (4(x-t))\right)
\end{aligned}
$$

Applying (54), we can get a solution of the third $\hat{C}_{2}^{(1)}$-flow,

$$
\tilde{q}=q_{1}\left(e_{11}-e_{44}\right)+q_{2}\left(e_{12}+4_{34}\right)+q_{3}\left(e_{13}-e_{24}\right)+q_{4} e_{14} .
$$

Using the algorithm in the proof of Proposition 5, we get a new solution of (5),

$$
\tilde{u}=\Delta * q=\tilde{u}_{1} e_{23}+u_{2} e_{14}
$$

where

$$
\begin{aligned}
& \tilde{u}_{1}=3 q_{1}^{\prime}+2 q_{2}+q_{1}^{2}, \\
& \tilde{u}_{2}=\left(q_{1}\right)_{x}^{(3)}+q_{2}^{\prime \prime}+q_{3}^{\prime}+q_{4}+2 q_{1} q_{3}-q_{2}^{2}-q_{1}^{\prime} q_{2}+q_{1} q_{2}^{\prime}+q_{1} q_{1}^{\prime \prime} .
\end{aligned}
$$

We use Theorem 12, and the formula for $\tilde{\pi}$, and a direct computation to see that

$$
\tilde{\gamma}(x, t)=\left(\begin{array}{cccc}
1 & 0 & 0 & 0 \\
-x & -1 & 0 & 0 \\
\frac{1}{2} x^{2} & x & 1 & 0 \\
-\left(\frac{1}{6} x^{3}+t\right) & -\frac{1}{2} x^{2} & -x & -1
\end{array}\right)\left(\begin{array}{l}
\xi_{1} \\
\xi_{2} \\
\xi_{3} \\
\xi_{4}
\end{array}\right)
$$


is a solution of the third Lagrangian curve flow of C-type on $\mathcal{M}_{4}$, where

$$
\begin{aligned}
& \xi_{1}=\frac{1}{y_{1}} \cosh ^{2}(x+t)+\left(1+\frac{y_{2}}{y_{3}}\right) \cos ^{2}(x-t)-\frac{y_{2}}{2 y_{3}} \sin (2(x-t))-1, \\
& \xi_{2}=-\frac{1}{4}\left(\cosh (2(x+t))+\left(1+\frac{y_{2}}{y_{3}}\right) \sin (2(x-t))-2 \frac{y_{2}}{y_{3}} \sin ^{2}(x-t)\right), \\
& \xi_{3}=\frac{1}{2}\left(\frac{1}{y_{1}} \cosh ^{2}(x+t)-\left(1+\frac{y_{2}}{y_{3}}\right) \cos ^{2}(x-t)+\frac{1}{2} \frac{y_{2}}{y_{3}} \sin (2(x-t))\right), \\
& \xi_{4}=-\frac{1}{4}\left(\cosh (2(x+t))-\left(1+\frac{y_{2}}{y_{3}}\right) \sin (2(x-t))+2 \frac{y_{2}}{y_{3}} \sin ^{2}(x-t)\right) .
\end{aligned}
$$

Next we give a Permutability formula for DTs of the $\hat{C}_{n}^{(1)}$ flows. The following Lemma follows from Lemma 4.

Lemma 5. Let $\alpha_{1}, \alpha_{2}$ be distinct real constants, $\pi_{1}, \pi_{2}$ projections of $\mathbb{R}^{2 n}$ satisfying $(48)$, and $\tau_{1}, \tau_{2}$ projections defined by

$$
\begin{cases}\operatorname{Im}\left(\tau_{1}\right)=k_{\alpha_{2}, \pi_{2}}\left(\alpha_{1}\right) \operatorname{Im}\left(\pi_{1}\right), & \operatorname{Ker}\left(\tau_{1}\right)=k_{\alpha_{2}, \pi_{2}}\left(-\alpha_{1}\right) \operatorname{Ker}\left(\pi_{1}\right), \\ \operatorname{Im}\left(\tau_{2}\right)=k_{\alpha_{1}, \pi_{1}}\left(\alpha_{2}\right) \operatorname{Im}\left(\pi_{2}\right), & \operatorname{Ker}\left(\tau_{2}\right)=k_{\alpha_{1}, \pi_{1}}\left(-\alpha_{2}\right) \operatorname{Ker}\left(\pi_{2}\right) .\end{cases}
$$

Then $\tau_{1}, \tau_{2}$ satisfy (48) and

$$
k_{\alpha_{2}, \tau_{2}} k_{\alpha_{1}, \pi_{1}}=k_{\alpha_{1}, \tau_{1}} k_{\alpha_{2}, \pi_{2}}
$$

It follows from Lemma 5 and Theorems 8 and 9 that we have

Theorem 13 (Permutability Formula for the $\hat{C}_{n}^{(1)}$-flows).

Let $F(x, t, \lambda)$ be the frame of the solution $q$ of the $(2 j-1)$-th $\hat{C}_{n}^{(1)}$-flow (14) with $F(0,0, \lambda)=$ $\mathrm{I}_{2 n}, \alpha_{i}, \pi_{i}, \tau_{i}$ for $i=1,2$ as in Lemma 5 . Then we have the following:

(1) Let $\tilde{V}_{i}=F\left(x, t, \alpha_{i}\right)^{-1}\left(\operatorname{Im} \pi_{i}\right)$, and $\tilde{W}_{i}=F\left(x, t,-\alpha_{i}\right)^{-1}\left(\operatorname{Ker} \pi_{i}\right)$, $\tilde{\pi}_{i}$ the projection onto $\tilde{V}_{i}$ along $\tilde{W}_{i}$ for $i=1,2$. Then

$$
\begin{aligned}
& q_{1}:=k_{\alpha_{1}, \pi_{1}} \bullet q=q+2 \alpha_{1}\left[e_{1,2 n}, \tilde{\pi}_{1}\right], \\
& q_{2}:=k_{\alpha_{2}, \pi_{2}} \bullet q=q+2 \alpha_{2}\left[e_{1,2 n}, \tilde{\pi}_{2}\right] .
\end{aligned}
$$

(2) $\quad k_{\alpha_{2}, \tau_{2}} \bullet\left(k_{\alpha_{1}, \pi_{1}} \bullet q\right)=k_{\alpha_{1}, \tau_{1}} \bullet\left(k_{\alpha_{2}, \pi_{2}} \bullet q\right)$.

(3) Let $\tilde{\tau}_{1}(x, t), \tilde{\tau}_{2}(x, t)$ be the projections defined by

$$
\begin{array}{ll}
\operatorname{Im}\left(\tilde{\tau}_{1}\right)=k_{\alpha_{2}, \tilde{\pi}_{2}}\left(\alpha_{1}\right) \operatorname{Im}\left(\tilde{\pi}_{1}\right), & \operatorname{Ker}\left(\tilde{\tau}_{1}\right)=k_{\alpha_{2}, \tilde{\pi}_{2}}\left(-\alpha_{1}\right) \operatorname{Ker}\left(\tilde{\pi}_{1}\right), \\
\operatorname{Im}\left(\tilde{\tau}_{2}\right)=k_{\alpha_{1}, \tilde{\tau}_{1}}\left(\alpha_{2}\right) \operatorname{Im}\left(\tilde{\pi}_{2}\right), & \operatorname{Ker}\left(\tilde{\tau}_{2}\right)=k_{\alpha_{1}, \tilde{\pi}_{1}}\left(-\alpha_{2}\right) \operatorname{Ker}\left(\tilde{\pi}_{2}\right) .
\end{array}
$$

Then we have

$$
\begin{aligned}
q_{12} & :=k_{\alpha_{2}, \tau_{2}} \bullet\left(k_{\alpha_{1}, \pi_{1}} \bullet q\right)=k_{\alpha_{1}, \tau_{1}} \bullet\left(k_{\alpha_{2}, \pi_{2}} \bullet q\right) \\
& =q_{1}+2 \alpha_{2}\left[e_{1,2 n}, \tilde{\tau}_{2}\right]=q_{2}+2 \alpha_{1}\left[e_{1,2 n}, \tilde{\tau}_{1}\right] .
\end{aligned}
$$

In particular, $q_{12}$ can be obtained algebraically from $\tilde{\pi}_{1}$ and $\tilde{\pi}_{2}$.

The Permutability Theorem 13 gives an algebraic formula for constructing $k$-solitons and their frames from $k 1$-solitons for the $\hat{C}_{n}^{(1)}$-flow. If $\tilde{F}$ is a frame of the $k$-soliton solution $\tilde{q}$ of $\hat{C}_{n}^{(1)}$-flow, then $\tilde{\gamma}=\tilde{F}(x, t, 0) e_{1}$ is a k-soliton solution of the Lagrangian curve flow of C-type and its Lagrangian curvature $\tilde{u}$ is a $k$-soliton of the $\hat{C}_{n}^{(1)}-\mathrm{KdV}$ flow. 


\section{Darboux Transforms for the $\hat{A}_{2 n-1}^{(2)}$-Hierarchy}

In this section, we construct Darboux transformations for the $\hat{A}_{2 n-1}^{(2)}, \hat{A}_{2 n-1}^{(2)}-\mathrm{KdV}$, and the Lagrangian curve flows of A type. We also give a Permutability formula for these Darboux transforms.

Let $\left(\hat{\mathbb{A}}_{2 n-1}^{(2)}\right)_{+}$denote the group of holomorphic maps $f: \mathbb{C} \rightarrow S L(2 n+1, \mathbb{C})$ satisfying the reality condition (7), i.e.,

$$
\overline{f(\bar{\lambda})}=f(\lambda), \quad f(-\lambda)^{t} S_{n} f(\lambda)=S_{n}
$$

and $\mathbb{R} \hat{\mathbb{A}}_{2 n-1}^{(2)}$ the group of rational maps $f: \mathbb{C} \rightarrow S L(2 n+1, \mathbb{C})$ satisfying (7) with $f(\infty)=\mathrm{I}$. Then the Lie algebras of $\left(\hat{\mathbb{A}}_{2 n-1}^{(2)}\right)_{+}$and $\mathbb{R} \hat{\mathbb{A}}_{2 n-1}^{(2)}$ are subalgebras of $\left(\hat{A}_{2 n-1}^{(2)}\right)_{+}$and $\left(\hat{A}_{2 n-1}^{(2)}\right)$ - respectively.

Please note that the second condition of (59) is equivalent to

$$
f^{-1}(\lambda)=f(-\lambda)_{s}
$$

where $A_{s}=S_{n}^{-1} A^{t} S_{n}$.

Please note that the restriction of the symplectic form $w$ to a linear subspace $V$ of $\mathbb{R}^{2 n}$ is non-degenerate if and only if $\mathbb{R}^{2 n}=V \oplus V^{\perp}$.

Lemma 6. Let $\pi$ be a projection. Then $\operatorname{Ker}(\pi)=(\operatorname{Im}(\pi))^{\perp}$ if and only if

$$
\pi=\pi_{s} .
$$

Lemma 7. Let $\pi$ be a projection of $\mathbb{R}^{2 n}$ satisfying (60), and $\alpha \in \mathbb{R} \backslash\{0\}$. Then $k_{\alpha, \pi}$ defined by (50) is in $\mathbb{R} \hat{\mathbb{A}}_{2 n-1}^{(2)}$.

Lemma 8. Let $\alpha \in \mathbb{R} \backslash 0$, $\pi$ a projection satisfying (60), and $f: \mathbb{C} \rightarrow G L(2 n, \mathbb{C})$ a meromorphic map, holomorphic at $\lambda=\alpha$ and $\lambda=-\alpha$, and satisfying (59). Let $\tilde{V}=f(\alpha)^{-1}(V)$, where $V=\operatorname{Im} \pi$. Then

(1) $\tilde{V}^{\perp}=f(-\alpha)^{-1}\left(V^{\perp}\right)$,

(2) assume that $\tilde{V} \cap \tilde{V}^{\perp}=0$, let $\tilde{\pi}$ be the projection onto $\tilde{V}$ along $\tilde{V}^{\perp}$, then

$$
\tilde{f}=k_{\alpha, \pi} f k_{\alpha, \tilde{\pi}}^{-1}
$$

is holomorphic at $\lambda=\alpha,-\alpha$ and satisfies (59).

Proof. Set $V=\operatorname{Im} \pi$. If $Y \in \tilde{V}^{\perp}$, then

$$
0=\omega\left(f(\alpha)^{-1} V, Y\right)=\omega\left(f(-\alpha)_{s} V, Y\right)=\omega(V, f(-\alpha) Y) .
$$

Hence $f^{-1}(-\alpha) Y \in V^{\perp}$, which implies that $f^{-1}(-\alpha)\left(\tilde{V}^{\perp}\right) \subset V^{\perp}$. Since they have the same dimension, $f^{-1}(-\alpha)\left(\tilde{V}^{\perp}\right)=V^{\perp}$. This proves (1).

By (51), we have

$$
\tilde{f}(\lambda)=\left(\mathrm{I}+\frac{2 \alpha}{\lambda-\alpha}(\mathrm{I}-\pi)\right) f(\lambda)\left(\mathrm{I}-\frac{2 \alpha}{\lambda+\alpha}(\mathrm{I}-\tilde{\pi})\right) .
$$

Please note that $\tilde{f}$ has a simple pole at $\lambda=\alpha$ and $\lambda=-\alpha$. The residue of $\tilde{f}$ at $\lambda=\alpha$ is $2 \alpha(\mathrm{I}-\pi) f(\alpha) \tilde{\pi}$, which is zero because $\operatorname{Im}(f(\alpha) \tilde{\pi})=f(\alpha)(\tilde{V})=V$ and $\operatorname{Ker}(\mathrm{I}-\pi)=V$. The residue of $\tilde{f}$ at $\lambda=-\alpha$ is $-2 \alpha \pi f(-\alpha)(\mathrm{I}-\tilde{\pi})$, which is zero because $\pi f(-\alpha) \tilde{V}^{\perp}=$ $\pi V^{\perp}=0$. Hence $\tilde{f}$ is holomorphic at $\lambda=\alpha,-\alpha$. Since both $f$ and $k_{\alpha, \pi}$ satisfies (59), $\tilde{f}$ satisfies (59). 
Using Lemma 8, Theorem 7 and a proof similar to the proof of Theorem 9, we obtain the following:

Theorem 14 (DT for the $\hat{A}_{2 n-1}^{(2)}$-hierarchy).

Let $c \in S p(2 n)$ be a constant, and $F(x, t, \lambda)$ be the frame of a solution $q$ of the $(2 j-1)$-th $\hat{A}_{2 n-1}^{(2)}$-flow (31) with $F(0,0, \lambda)=c$, and $\pi$ a projection satisfying (60). Given $\alpha \in \mathbb{R} \backslash\{0\}$, let

$$
\tilde{V}(x, t)=F(x, t, \alpha)^{-1}(V), \quad \text { where } V=\operatorname{Im} \pi \text {. }
$$

Then

(1) there exists an open neighborhood $\mathcal{O}$ of $(0,0)$ in $\mathbb{R}^{2}$ such that $\mathbb{R}^{2 n}=\tilde{V}(x, t) \oplus \tilde{V}(x, t)^{\perp}$ for all $(x, t) \in \mathbb{R}^{2}$,

(2) let $\tilde{\pi}(x, t)$ be the projection onto $\tilde{V}(x, t)$ along $\tilde{V}^{\perp}(x, t)$, then

$$
\tilde{q}=q+\alpha\left[e_{1,2 n-1}+e_{2,2 n}, \tilde{\pi}\right]
$$

is a solution of (31) defined on $\mathcal{O}$ and

$$
\tilde{F}(x, t, \lambda)=k_{\alpha, \pi}(\lambda) F(x, t, \lambda) k_{\alpha, \tilde{\pi}(x, t)}^{-1}(\lambda)
$$

is a frame of $\tilde{q}$.

In particular, if $F$ satisfies $F(0,0, \lambda)=\mathrm{I}_{2 n}$, then we have

$$
k_{\alpha, \pi} \bullet q=q+\alpha\left[e_{1,2 n-1}+e_{2,2 n}, \tilde{\pi}\right] .
$$

Theorem 14 can be reformulated as follows:

Theorem 15. Let $q$ be a solution of (31), $\lambda \in \mathbb{R} \backslash 0$, and $B_{q, \lambda}$ the following linear system

$$
B_{q, \lambda}\left\{\begin{array}{l}
y_{x}=-(\beta \lambda+b+q) y, \\
y_{t}=-\left(Q^{2 j-1}(q, \lambda)\right)+y
\end{array}\right.
$$

where $\beta=\frac{1}{2}\left(e_{1,2 n-1}+e_{2,2 n}\right)$. Then we have the following:

(1) (63) is solvable.

(2) Let $\left\{v_{1}, \ldots, v_{2 n}\right\}$ be a basis of $\mathbb{R}^{2 n}$ such that $w\left(v_{i}, v_{n+j}\right)=0$ for all $1 \leq i, j \leq n$. Let $y_{i}$ be the solution of $D_{q, \alpha}$ with initial data $y_{i}(0,0)=v_{i}$, and $y_{n+i}$ the solution of $D_{q,-\alpha}$ with $y_{n+i}(0,0)=v_{n+i}$ for $1 \leq i \leq n$. Let $\tilde{V}_{1}(x, t)$ be the span of $y_{1}(x, t), \ldots, y_{n}(x, t)$, and $\tilde{V}_{2}(x, t)$ the span of $y_{n+1}(x, t), \ldots, y_{2 n}(x, t)$. Then

(a) $\tilde{V}_{2}(x, t)=\tilde{V}_{1}(x, t)^{\perp}$ for all $(x, t) \in \mathbb{R}^{2}$ and $i=1,2$,

(b) there is an open subset $\mathcal{O}$ of $(0,0)$ such that $\tilde{V}_{1}(x, t) \cap \tilde{V}_{2}(x, t)=0$,

(c) $\tilde{q}$ defined by (61) is a solution of (31) defined on $\mathcal{O}$, where $\tilde{\pi}$ is the projection onto $\tilde{V}_{1}(x, t)$ along $\tilde{V}_{2}(x, t)$.

Bäcklund transformations for the $\hat{A}_{2 n-1}^{(2)}$-flows are obtained in the similar way as for the $\hat{C}_{n}^{(1)}$-flows.

As a consequence of Proposition 9 and Theorem 14, we obtain the following:

\section{Theorem 16 (DT for $\left.\hat{A}_{2 n-1}^{(2)}-\mathrm{KdV}(33)\right)$.}

Let $E$ be a frame of a solution $u$ of (34), $\triangle: \mathbb{R}^{2} \rightarrow N_{n}^{+}$a solution of $\triangle_{t} \triangle^{-1}=\xi_{j}(u)$, and $q=\triangle^{-1} * u$, where $\xi_{j}(u)$ is defined by (33). Let $\pi$ be a projection satisfying (60), and $k_{\alpha, \pi}$ defined by (50), and $\tilde{V}(x, t)=\triangle^{-1}(x, t) E^{-1}(x, t, \alpha)(\operatorname{Im} \pi)$. Then

(1) there exists an open subset containing $(0,0)$ such that $\mathbb{R}^{2 n}=\tilde{V}(x, t) \oplus \tilde{V}^{\perp}(x, t)$, 
(2) let $\tilde{\pi}(x, t)$ denote the projection onto $\tilde{V}(x, t)$ along $\tilde{V}(x, t)^{\perp}, \tilde{q}$ defined by (61), and $\widetilde{\triangle}$ : $\mathbb{R}^{2} \rightarrow N_{n}^{+}$such that $\tilde{\Delta} * \tilde{q}$ is $V_{n}$-valued. Then $\tilde{u}=\tilde{\triangle} * \tilde{q}$ is a new solution of (34) and

$$
\tilde{E}=k_{\alpha, \pi} E \triangle k_{\alpha, \tilde{\pi}}^{-1} \tilde{\triangle}^{-1}
$$

is a frame of $\tilde{u}$.

Theorems 14 and 6 (iii) give the following:

Theorem 17 (DT for Lagrangian curve flows of A-type).

Let $\gamma$ be a solution of the Lagrangian curve flow (40) of A-type, and $g(\cdot, t), u(\cdot, t)$ the Lagrangian frame and Lagrangian curvature along $\gamma(\cdot, t)$. Let $E$ be the frame of the solution $u$ of (31) satisfying $E(0,0, \lambda)=g(0,0)$. Let $\triangle, \alpha, \pi, \tilde{\pi}$ be as in Theorem 16. Then

$$
\tilde{\gamma}=(2 \pi-\mathrm{I}) g \triangle(2 \tilde{\pi}-\mathrm{I}) e_{1}
$$

is a new solution of (40) and its Lagrangian curvature $\tilde{u}$ is a solution of (31).

\section{Example 12. 1-soliton solutions of A-type}

Please note that $u=0$ is the trivial solution of the third $\hat{A}_{2 n-1}^{(2)}$-flow with frame $F(x, t, \lambda)=$ $\exp \left(x J_{B}(\lambda)+t J_{B}^{3}(\lambda)\right)$. By Theorem 6 (iii),

$$
\gamma(x, t)=F(x, t, 0) e_{1}=\exp \left(b x+b^{3} t\right) e_{1}
$$

is the Lagrangian curve flow (39) with zero Lagrangian curvature and

$$
g(x, t)=\exp \left(b x+b^{3} t\right)
$$

as its Lagrangian frame.

Please note that the linear system $B_{q, \lambda}$ given by (63) for $q=0$ is

$$
B_{0, \lambda}\left\{\begin{array}{l}
y_{x}=-J_{B} y, \\
y_{t}=-J_{B}^{3} y .
\end{array}\right.
$$

Since

$$
J_{B}^{2 n}=\lambda J_{B}, \quad\left(J_{B}^{3}\right)^{2 n}=\lambda^{3} J_{B}^{3},
$$

the solution of $B_{0, \lambda}$ for any given initial data can be written down explicitly. Hence Theorem 15 gives an algorithm to compute explicit formula for 1-solitons $\tilde{q}$ and its frame for the third $\hat{A}_{2 n-1}^{(2)}$ flow. Theorem 17 gives the corresponding 1-soliton solution $\tilde{\gamma}$ of the third Lagrangian curve flow of A-type and the Lagrangian curvature $\tilde{u}$ of $\tilde{\gamma}$ is a 1-soliton solution of the third $\hat{A}_{2 n-1}^{(2)}-K d V$ flow.

Next we give the Permutability formula. First it follows from Lemma 8 that we have the following:

Lemma 9. Let $\alpha_{1}, \alpha_{2} \in \mathbb{R} \backslash\{0\}$ such that $\left|\alpha_{1}\right| \neq\left|\alpha_{2}\right|$, and $\pi_{i}$ projections of $\mathbb{R}^{2 n}$ satisfying $\operatorname{Ker} \pi_{i}=\left(\operatorname{Im} \pi_{i}\right)^{\perp}$. Then $\tilde{V}_{1}=k_{\alpha_{2}, \pi_{2}}\left(\alpha_{1}\right)\left(\operatorname{Im} \pi_{1}\right)$ and $\tilde{V}_{2}=k_{\alpha_{1}, \pi_{1}}\left(\alpha_{2}\right)\left(\operatorname{Im} \pi_{2}\right)$ are nondegenerate, and

$$
k_{\alpha_{1}, \tau_{1}} k_{\alpha_{2}, \pi_{2}}=k_{\alpha_{2}, \tau_{2}} k_{\alpha_{1}, \pi_{1}}
$$

where $\tau_{i}$ is the projection onto $\tilde{V}_{i}$ along $\tilde{V}_{i}^{\perp}$ for $i=1,2$.

Similarly, Lemma 9, Theorems 8 and 14 give the following:

Theorem 18. [Permutability for DTs of the $\hat{A}_{2 n-1}^{(2)}$-flow] 
Let $\alpha_{i}, \pi_{i}, \tau_{i}$ be as in Lemma 9 for $i=1,2$. Let $F$ be the frame of a solution $q$ of the $(2 j-1)$-th $\hat{A}_{2 n-1}^{(2)}$-flow with $F(0,0, \lambda)=\mathrm{I}, \tilde{V}_{i}(x, t)=F\left(x, t, \alpha_{i}\right)^{-1}\left(\operatorname{Im} \pi_{i}\right)$, and $\tilde{\pi}_{i}(x, t)$ the projection onto $\tilde{V}_{i}(x, t)$ along $\tilde{V}_{i}(x, t)^{\perp}$. Let $\tilde{W}_{1}=k_{\alpha_{2}, \tilde{\pi}_{2}}\left(\alpha_{1}\right)\left(\operatorname{Im} \tilde{\pi}_{1}\right), \tilde{W}_{2}=k_{\alpha_{1}, \tilde{\pi}_{1}}\left(\alpha_{2}\right)\left(\operatorname{Im} \tilde{\pi}_{2}\right)$, and $\tilde{\tau}_{i}$ be the projection onto, $\tilde{W}_{i}$ along $\tilde{W}_{i}^{\perp}$. Then we have

$$
\begin{aligned}
& q_{i}:=k_{\alpha_{i}, \pi_{i}} \bullet q=q+\alpha_{i}[\beta, \tilde{\pi}], \quad i=1,2, \\
& k_{\alpha_{1}, \tau_{1}} \bullet\left(k_{\alpha_{2}, \pi_{2}} \bullet q\right)=k_{\alpha_{2}, \tau_{2}} \bullet\left(k_{\alpha_{1}, \pi_{1}} \bullet q\right), \\
& q_{12}:=k_{\alpha_{1}, \tau_{1}} \bullet\left(k_{\alpha_{2}, \pi_{2}} \bullet q\right)=q_{1}+\alpha_{2}\left[\beta, \tilde{\tau}_{2}\right]=q_{2}+\alpha_{1}\left[\beta, \tilde{\tau}_{1}\right],
\end{aligned}
$$

where $\beta=e_{1,2 n-1}+e_{2,2 n}$.

The Permutability Theorem 18 gives an algebraic formula to construct $k$-solitons of the $(2 j-1)$-th $\hat{A}_{2 n-1}^{(2)}$-flow and their frames from $k 1$-solitons of the $(2 j-1)$-th $\hat{A}_{2 n-1}^{(2)}$ flow. If $\tilde{F}$ is a frame of the $k$-soliton solution $\tilde{q}$ of $\hat{A}_{2 n-1}^{(2)}$-flow, then $\tilde{\gamma}=\tilde{F}(x, t, 0) e_{1}$ is a k-soliton solution of the Lagrangian curve flow of A-type and its Lagrangian curvature $\tilde{u}$ is a $k$-soliton of the $\hat{A}_{2 n-1}^{(2)}-\mathrm{KdV}$ flow.

\section{Scaling Transforms}

In this section, we construct scaling transforms and give relations between DTs and scaling transforms for the $\hat{C}_{n}^{(1)}$-flows and $\hat{A}_{2 n-1}^{(2)}$-flows.

Theorem 19. Let $\alpha_{i}, \pi_{i}, \tau_{i}$ as in Lemma 5 (9 resp.), and $F(x, t, \lambda)$ the frame of the solution $q$ of the $(2 j-1)$-th $\hat{C}_{n}^{(1)}$-flow (14) $\left(\hat{A}_{2 n-1}^{(2)}\right.$-flow (31) resp.) with $F(0,0, \lambda)=I_{2 n+1}$. Let $r \in \mathbb{R} \backslash\{0\}$, and

$$
\Gamma(r)=\operatorname{diag}\left(1, r, \ldots, r^{2 n-1}\right) .
$$

Then

(1) $\quad(r \odot q)(x, t)=r \Gamma(r)^{-1} q\left(r x, r^{2 j-1} t\right) \Gamma(r)$ is a solution of the $(2 j-1)$-th $\hat{C}_{n}^{(1)}$-flow (the $\hat{A}_{2 n-1}^{(2)}$-flow resp.),

(2) for the $\hat{C}_{n}^{(1)}$ case,

$$
(r \odot F)(x, t, \lambda):=\Gamma(r)^{-1} F\left(r x, r^{2 j-1} t, r^{-2 n} \lambda\right) \Gamma(r)
$$

is the frame of the solution $r \odot q$ of the $\hat{C}_{n}^{(1)}$-flow (14),

(3) for the $\hat{A}_{2 n-1}^{(2)}$-case,

$$
(r \odot F)(x, t, \lambda):=\Gamma(r)^{-1} F\left(r x, r^{2 j-1} t, r^{-(2 n-1)} \lambda\right) \Gamma(r)
$$

is the frame of the solution $r \odot q$ of the $\hat{A}_{2 n-1}^{(2)}$-flow (31).

Proof. First we prove the Theorem for the $\hat{C}_{n}^{(1)}$-flows. Set $\hat{F}(x, t, \lambda)=\Gamma(r)^{-1} F\left(r x, r^{2 j-1} t\right.$, $\left.r^{-2 n} \lambda\right)$. Please note that

$$
r \Gamma(r)^{-1}\left(e_{1,2 n} r^{-2 n} \lambda+b\right) \Gamma(r)=e_{1,2 n} \lambda+b=J(\lambda) .
$$

Since $F$ is a frame of $q, F^{-1} F_{x}=J+q$. direct computation implies that

$$
\hat{F}^{-1} \hat{F}_{x}=J+r q\left(r x, r^{2 j-1} t, r^{-2 n} \lambda\right) .
$$

Let $P(x, t, \lambda)=P(q(x, t), \lambda)$ be the solution of (10). So $P_{x}+[J+q, P]=0$. Set

$$
\hat{P}(x, t, \lambda)=r \Gamma(r)^{-1} P\left(q\left(r x, r^{2 j-1} t\right), r^{-2 n} \lambda\right) \Gamma(r) .
$$


We use (65) and a direct computation to see that

$$
\hat{P}_{x}+[J+r \odot q, \hat{P}]=0 .
$$

This shows that $\hat{P}=P(r \odot q, \lambda)$. A direct computation implies that

$$
\begin{aligned}
\hat{F}^{-1} \hat{F}_{t} & =\Gamma(r)^{-1}\left(r^{2 j-1} P^{2 j-1}\left(r x, r^{2 j-1} t, r^{-2 n} \lambda\right)_{+} \Gamma(r)\right) \\
& =\Gamma(r)^{-1}\left(r P\left(r x, r^{2 j-1} t, r^{-2 n} \lambda\right)\right)_{+}^{2 j-1} \Gamma(r)=\left(\hat{P}^{2 j-1}\right)_{+} \\
& =\left(P^{2 j-1}(r \odot q, \lambda)\right)_{+} .
\end{aligned}
$$

It follows from Proposition 4 that $r \odot q$ is a solution of (14) and $\hat{F}$ is a frame of $r \odot q$. This proves (1) and (2) for the $\hat{C}_{n}^{(1)}$-hierarchy.

Similar proof gives (1) and (3) for the $\hat{A}_{2 n-1}^{(2)}$-hierarchy.

It follows from Theorem 19 (2) and Theorem 6 (iii) that we have the following:

Corollary 4. Let $c \in \mathbb{R} \backslash 0$, and $\gamma$ a solution of the $(2 j-1)$-th Lagrangian curve flow of C-type or A-type. Then

$$
(c \odot \gamma)(x, t):=\Gamma(c) \gamma(c x, c t)
$$

is again a solution, where $\Gamma(c)$ is defined by (64).

In particular, let $\tilde{\gamma}$ be the solution of the third Lagrangian curve flow on $\mathcal{M}_{4}$ constructed in Example 11. Then $c \odot \tilde{\gamma}$ is also a solution for all $c \in \mathbb{R} \backslash 0$.

Corollary 5. Let $u=\sum_{i=1}^{n} u_{i} e_{n+1-i, n+i}$ be a solution of the $(2 j-1)-t h \hat{C}_{n}^{(1)}-K d V$ flow (22) $\left(\hat{A}_{2 n-1}^{(2)}-K d V\right.$ flow (34) resp.), $r \in \mathbb{R} \backslash\{0\}, \Gamma(r)$ as in (7). Then we have the following:

(1) $\quad r \odot u=\sum_{i=1}^{n} r^{2 i} u_{i}\left(r x, r^{2 j-1} t\right) e_{n+1-i, n-i}$ is a solution of the $(2 j-1)-t h \hat{C}_{n}^{(1)}-K d V$ flow (22) $\left(\hat{A}_{2 n-1}^{(2)}-K d V\right.$ flow (34) resp.).

(2) If $E(x, t, \lambda)$ is a frame of the solution $u$ of $(22)$, then

$$
(r \odot E)(x, t, \lambda):=\Gamma(r)^{-1} E\left(r x, r^{2 j-1} t, r^{-2 n} \lambda\right) \Gamma(r)
$$

is a frame of $r \odot u$.

(3) If $E(x, t, \lambda)$ is a frame of the solution $u$ of (34), then

$$
(r \odot E)(x, t, \lambda):=\Gamma(r)^{-1} E\left(r x, r^{2 j-1} t, r^{-(2 n-1)} \lambda\right) \Gamma(r)
$$

is a frame of $r \odot u$.

Corollary 6. $r \odot u$ defines an action of the multiplicative group $\mathbb{R}^{+}$on the space of solutions of (22) ((34) resp.).

Next we give a relation between the scaling transforms and Darboux transforms. First we need a Lemma.

Lemma 10. Let $r \in \mathbb{R} \backslash 0, \Gamma(r)$ defined by (64), and $A_{s}=S_{n}^{-1} A^{t} S_{n}$ as before. Then

(1) $\Gamma(r)_{s}=r^{2 n+1} \Gamma(r)^{-1}$,

(2) let $\pi$ be a projection of $\mathbb{R}^{2 n}$, and $\hat{\pi}=\Gamma(r) \pi \Gamma(r)^{-1}$, then

(a) if $\pi_{s}=\pi$, then $\hat{\pi}_{s}=\hat{\pi}$,

(b) if $\pi_{s}=\mathrm{I}-\pi$, then $\hat{\pi}_{s}=\mathrm{I}-\hat{\pi}$.

Proof. It is clear that $\Gamma(r) S_{n} \Gamma(r)=r^{2 n+1} S_{n}$, which gives (1). (2) follows from (1). 
It follows from Lemma 10, the formulas for $r \odot q$ in Theorem 19 and (55), (62) that we have the following.

Theorem 20. Let $r, \alpha \in \mathbb{R} \backslash 0, \Gamma(r)$ as in (64), $\pi$ a projection of $\mathbb{R}^{2 n}$, and $\hat{\pi}=\Gamma(r) \pi \Gamma(r)^{-1}$.

(1) If $q$ is a solution of the $(2 j-1)$-th $\hat{C}_{n}^{(1)}$-flow (14) and $\pi$ satisfies $\pi_{s}=\mathrm{I}-\pi$, then

$$
k_{r-2 n, \hat{\pi}} \bullet q=r^{-1} \odot\left(k_{1, \pi} \bullet(r \odot q)\right) .
$$

(2) If $q$ is a solution of the $(2 j-1)$-th $\hat{A}_{2 n-1}^{(2)}$-flow (31) and $\pi$ satisfies $\pi_{s}=\pi$, then

$$
k_{r^{-(2 n-1), \hat{\pi}}} \bullet q=r^{-1} \odot\left(k_{1, \pi} \bullet(r \odot q)\right) .
$$

\section{Bi-Hamiltonian Structure}

The existence of a bi-Hamiltonian structure and using it to generate the hierarchy are two of the well-known properties for soliton hierarchies (cf. [11,55,56]). In this section, we use the linear operator $P_{u}$ defined in Definition 4 to write down the bi-Hamiltonian structure for the $\hat{C}_{n}^{(1)}-\mathrm{KdV}$ and $\hat{A}_{2 n-1}^{(2)}-\mathrm{KdV}$. The pull back of this bi-Hamiltonian structure to $\mathcal{M}_{2 n}$ via the Lagrangian curvature map $\Psi$ gives the bi-Hamiltonian structure for the Lagrangian curve flows of $C$ and $A$-type.

Let

$$
\langle\xi, \eta\rangle=\oint \operatorname{tr}(\xi \eta) \mathrm{d} x
$$

denote the standard $L^{2}$ inner product on $C^{\infty}\left(S^{1}, \operatorname{sl}(2 n, \mathbb{R})\right)$.

The bi-Hamiltonian structure on $C^{\infty}\left(S^{1}, \mathcal{B}_{n}^{+}\right)$for the $\hat{C}_{n}^{(1)}$ and $\hat{A}_{2 n-1}^{(2)}$ hierarchies given in [11] is

$$
\begin{aligned}
& \left\{F_{1}, F_{2}\right\}_{1}^{\wedge}(q)=\left\langle\left[\beta, \nabla F_{1}(q)\right], \nabla F_{2}(q)\right\rangle, \\
& \left\{F_{1}, F_{2}\right\}_{2}^{\wedge}(q)=\left\langle\left[\partial_{x}+b+q, \nabla F_{1}(q)\right], \nabla F_{2}(q)\right\rangle,
\end{aligned}
$$

where

$$
\beta= \begin{cases}e_{1,2 n}, & \text { for } \hat{C}_{n}^{(1)}, \\ \frac{1}{2}\left(e_{1,2 n-1}+e_{2,2 n}\right), & \text { for } \hat{A}_{2 n-1}^{(2)} .\end{cases}
$$

Using the same proof as in [49], we see that the bi-Hamiltonian structure is invariant under the gauge action of the group $C^{\infty}\left(S^{1}, N_{n}^{+}\right)$, i.e., if $F_{1}, F_{2}$ are invariant under the gauge action, then $\left\{F_{1}, F_{2}\right\}_{i}^{\wedge}$ is also invariant for $i=1,2$. Since $C^{\infty}\left(S^{1}, V_{n}\right)$ is the orbit space of this gauge action, we can identify functionals $F$ on $C^{\infty}\left(S^{1}, V_{n}\right)$ with invariant functionals $\hat{F}$ on $C^{\infty}\left(S^{1}, \mathcal{B}_{n}^{+}\right)$, where

$$
\hat{F}(\triangle * u)=F(u)
$$

Hence

$$
\left\{F_{1}, F_{2}\right\}_{i}(u)=\left\{\hat{F}_{1}, \hat{F}_{2}\right\}_{i}^{\wedge}(u)
$$

are Poisson structures on $C^{\infty}\left(S^{1}, V_{n}\right)$ for $i=1,2$.

Given a functional $F: C^{\infty}\left(S^{1}, V_{n}\right) \rightarrow \mathbb{R}$, let $\nabla F(u)$ be the unique map from $S^{1} \rightarrow$ $V_{n}^{t}$ satisfying

$$
\mathrm{d} F_{u}(v)=\langle\nabla F(u), v\rangle=\oint \operatorname{tr}(\nabla F(u) v) \mathrm{d} x
$$

for all $v \in C^{\infty}\left(S^{1}, V_{n}\right)$. 
Again we use the same proof as in $[49,50]$ to write $\{,\}_{i}$ in terms of the linear operator $P_{u}$ :

$$
\begin{aligned}
& \left\{F_{1}, F_{2}\right\}_{1}(u)=\left\langle\left[\beta, P_{u}\left(\nabla F_{1}(u)\right)\right], P_{u}\left(\nabla F_{2}(u)\right)\right\rangle, \\
& \left\{F_{1}, F_{2}\right\}_{2}(u)=\left\langle\left[\partial_{x}+b+u, P_{u}\left(\nabla F_{1}(u)\right)\right], P_{u}\left(\nabla F_{2}(u)\right)\right\rangle,
\end{aligned}
$$

where $\beta$ is given by (68) These give a bi-Hamiltonian structure for the $\hat{C}_{n}^{(1)}-\mathrm{KdV}$ flows.

The first bracket is always zero and $\{,\}_{2}$ is a Poisson structure for the $\hat{A}_{2 n-1}^{(2)}$-hierarchy. There is a standard way (cf. [56]) to generate a sequence of compatible invariant Poisson structures $\{,\}_{j}^{\wedge}, j \geq 1$ on $C^{\infty}\left(S^{1}, \mathcal{B}_{n}^{+}\right)$. It can be checked that the induced structure $\{,\}_{2 i+1}$ on $C^{\infty}\left(S^{1}, V_{n}\right)$ is always zero for the $\hat{A}_{2 n-1}^{(2)}-\mathrm{KdV}$ hierarchy, but $\{,\}_{2 i}$ are non-trivial Poisson structure. So $\left(\{,\}_{2},\{,\}_{4}\right)$ gives a bi-Hamiltonian structure for the $\hat{A}_{2 n-1}^{(2)}-\mathrm{KdV}$ flows. Since the formulas are tedious and do not give us useful information, we omit the discussion of $\{,\}_{4}$ for the $\hat{A}_{2 n-1}^{(2)}$-KdV hierarchy.

Since $\left[\partial_{x}+b+u, P_{u}\left(\nabla F_{1}(u)\right)\right]$ is in $C^{\infty}\left(S^{1}, V_{n}\right)$ and $\pi_{0}\left(P_{u}\left(\nabla F_{2}(u)\right)=\nabla F_{2}(u)\right.$, we have

$$
\left\{F_{1}, F_{2}\right\}_{2}(u)=\left\langle\left[\partial_{x}+b+u, P_{u}\left(\nabla F_{1}(u)\right)\right], \nabla F_{2}(u)\right\rangle .
$$

So the Hamiltonian flow for a functional $F$ with respect to $\{,\}_{2}$ is

$$
u_{t}=\left[\partial_{x}+b+u, P_{u}(\nabla F(u))\right] .
$$

The following results can be proved by a similar computation as in [49] for the $\hat{A}_{n}^{(1)}$ KdV hierarchy:

Theorem 21. Set

$$
\begin{aligned}
F_{2 j-1}(u) & =-\oint \operatorname{tr}\left(P_{2 j-1,-1}(u) e_{1,2 n}\right) \mathrm{d} x \\
H_{2 j-1}(u) & =-\frac{1}{2} \oint \operatorname{tr}\left(Q_{2 j-1,-1}(u)\left(e_{1,2 n+1}+e_{2,2 n}\right)\right) \mathrm{d} x .
\end{aligned}
$$

Then we have

$$
\nabla F_{2 j-1}(u)=\pi_{0}\left(P_{2 j-1,0}(u)\right), \quad \nabla H_{2 j-1}(u)=\pi_{0}\left(Q_{2 j-1,0}(u)\right),
$$

where $\pi_{0}$ is the projection onto $V_{n}^{t}$ defined by (44). Moreover, we also have:

(i) The Hamiltonian equation for $F_{2 j-1}\left(H_{2 j-1}\right.$ resp.) with respect to $\{,\}_{2}$ is the $(2 j-1)$-th $\hat{C}_{n}^{(1)}-K d V\left(\hat{A}_{2 n-1}^{(2)}-K d V\right.$ resp.) flow for $j \geq 1$.

(ii) The Hamiltonian equation for $F_{2(n+j)-1}$ with respect to $\{,\}_{1}$ is the $(2 j-1)-$ th $\hat{C}_{n}^{(1)}-K d V$ flow for $j>n$.

Remark 3. The bi-Hamiltonian structure on $C^{\infty}\left(S^{1}, V_{1}\right)$ for the $\hat{C}_{1}^{(1)}-K d V$ hierarchy is the standard bi-Hamiltonian structure for the KdV hierarchy (cf. [52]).

\section{Example 13. Bi-Hamiltonian structure for the $\hat{C}_{2}^{(1)}-\mathrm{KdV}$ hierarchy}


Let $u=u_{1} e_{23}+u_{2} e_{14}, \xi=\xi_{1} e_{32}+\xi_{2} e_{41}, \eta=\eta_{1} e_{32}+\eta_{2} e_{41}, C=\left(C_{i j}\right)=P_{u}(\xi)$, and $D=\left(D_{i j}\right)=P_{u}(\eta)$. We use Example 9 to write down the following Hamiltonian structures:

$$
\begin{aligned}
& \left\{F_{1}, F_{2}\right\}_{1}(u)=\left\langle\left[e_{14}, C\right], D\right\rangle \\
& =\oint\left(3 \xi_{2}^{\prime \prime \prime}+4 \xi_{1}^{\prime}-u_{1} \xi_{2}^{\prime}\right) \eta_{2}+4 \xi_{2}^{\prime} \eta_{1}+u_{1} \xi_{2} \eta_{2}^{\prime} \mathrm{d} x, \\
& =\oint\left(3 \xi_{2}^{\prime \prime \prime}+4 \xi_{1}^{\prime}-2 u_{1} \xi_{2}^{\prime}-u_{1}^{\prime} \xi_{2}\right) \eta_{2}+4 \xi_{2}^{\prime} \eta_{1} \mathrm{~d} x, \\
& \left\{F_{1}, F_{2}\right\}_{2}(u)=\left\langle\left[\partial_{x}+b+u, C\right], D\right\rangle \\
& \quad=\oint\left(C_{14}^{\prime}-2 u_{2} C_{11}\right) \eta_{2}+\left(C_{23}^{\prime}+2 C_{13}+u_{1} \xi_{1}^{\prime}\right) \eta_{1} \mathrm{~d} x,
\end{aligned}
$$

where $C_{i j}$ 's are written in terms of $\xi_{1}$ and $\xi_{2}$ as in Example 9.

\section{Example 14. Conservation laws for the $\hat{C}_{n}^{(1)}-\mathrm{KdV}$ hierarchy}

Let

$$
f_{2 j-1}(u)=\operatorname{tr}\left(P_{2 j-1,-1}(u) e_{1,2 n}\right)
$$

denote the density of $F_{2 j-1}$.

(1) For $n=2$, we have

$$
f_{1}=u_{1}, f_{3}=u_{2}+\frac{1}{8} u_{1}^{2}, f_{5}=-\frac{1}{32} u_{1}^{3}+u_{1} u_{2}-\frac{3}{32} u_{1} u_{1}^{\prime \prime} .
$$

(2) For general $n$, the first two densities of conservation laws are

$$
f_{1}=u_{1}, \quad f_{3}=u_{2}+\frac{2 n-3}{4 n} u_{1}^{2} .
$$

Example 15. Conservation laws for the $\hat{A}_{2 n-1}^{(2)}-\mathrm{KdV}$ hierarchy

Let

$$
h_{2 j-1}(u)=\frac{1}{2} \operatorname{tr}\left(Q_{2 j-1,-1}(u)\left(e_{1,2 n+1}+e_{2,2 n}\right)\right) .
$$

(1) For $n=2$, we have

$$
h_{1}=u_{1}, \quad h_{3}=u_{2}, \quad h_{5}=\frac{1}{3}\left(\frac{2}{3} u_{1}\left(u_{1}\right)_{x x}-4 u_{1} u_{2}-\frac{4}{9} u_{1}^{3}\right) .
$$

(2) For general $n$, the first two densities of conservation laws are

$$
h_{1}=u_{1}, \quad h_{3}=u_{2}+\frac{n-2}{2 n-1} u_{1}^{2} .
$$

Example 16. Hamiltonian flows for $F_{3}$ and $H_{3}$

A simple computation implies that $\nabla F_{3}(u)=\frac{1}{4} u_{1} e_{32}+e_{41}$, where $u=u_{1} e_{23}+u_{2} e_{14}$.

We use notations and formulas as in Example 9 to compute $P_{u}\left(\nabla F_{3}(u)\right)$ and obtain

$$
\begin{aligned}
& C_{11}=-\frac{3}{8} u_{1}^{\prime}, \quad C_{13}=-\frac{3}{8}\left(u_{1}\right)_{x}^{(3)}+u_{2}^{\prime}, \\
& C_{14}=-\frac{3}{8}\left(u_{1}\right)_{x}^{(4)}+\left(u_{2}\right)_{x x}+\frac{3}{8} u_{1}\left(u_{1}\right)_{x x}-\frac{3}{4} u_{1} u_{2}, \\
& C_{23}=-\frac{1}{2}\left(u_{1}\right)_{x x}+u_{2}+\frac{1}{4} u_{1}^{2} .
\end{aligned}
$$

The Hamiltonian flow of $F_{3}$ with respect to $\{,\}_{2}$ is

$$
u_{t}=\left[\partial_{x}+b+u, P_{u}\left(\nabla F_{3}(u)\right)\right] .
$$


We use the formula for $P_{u}\left(\nabla F_{3}(u)\right)$ to compute directly and see that (69) is the following system for $u_{1}, u_{2}$,

$$
\left\{\begin{array}{l}
\left(u_{1}\right)_{t}=C_{23}^{\prime}+2 C_{13}+\frac{1}{4} u_{1} u_{1}^{\prime} \\
\left(u_{2}\right)_{t}=C_{14}^{\prime}-2 u_{2} C_{11}
\end{array}\right.
$$

Substitute $C_{i j}$ into the above equation to see that it is (5).

Similarly, we use the same notations and formulas as in Example 9 to compute $P_{u}\left(\nabla H_{3}(u)\right)$. Here $\nabla H_{3}(u)=e_{32}$. We see thatbe

$$
C_{11}=0, \quad C_{13}=u_{2}^{\prime}, \quad C_{14}=u_{2}^{\prime \prime}-u_{1} u_{2}, \quad C_{23}=u_{2}
$$

So the Hamiltonian flow for $H_{3}$ with respect to $\{,\}_{2}$ written in terms of $u_{1}, u_{2}$ is (6).

Remark 4. We use the pullback $\{,\}_{i}^{\wedge}$ of the Poisson structures $\{,\}_{i}$ on $C^{\infty}\left(S^{1}, V_{n}\right)$ by the Lagrangian curvature map $\Psi$ for $i=1,2$, to get a bi-Hamiltonian structure on $\mathcal{M}_{2 n}$. In other words, given a functional $F_{i}$ on $C^{\infty}\left(S^{1}, V_{n}\right)$, let

$$
\hat{F}=F \circ \Psi: \mathcal{M}_{2 n} \rightarrow \mathbb{R}
$$

be functionals on $\mathcal{M}_{2 n}$. Then

$$
\{\hat{F}, \hat{G}\}_{i}^{\wedge}(\gamma)=\{F, G\}_{i}(\Psi(\gamma)), \quad i=1,2
$$

are the pullback bi-Hamiltonian on $\mathcal{M}_{2 n}$. As a consequence of Theorem 21, we have the following:

(1) The Lagrangian curve flow (39) and (40) are Hamiltonian flows for the Hamiltonians

$$
\hat{F}_{2 j-1}:=F_{2 j-1} \circ \Psi, \quad \hat{H}_{2 j-1}(u):=H_{2 j-1} \circ \Psi
$$

with respect to $\{,\}_{2}^{\wedge}$ respectively.

(2) The Lagrangian curve flows of C-type (A-type resp.) are commuting Hamiltonian flows on $\mathcal{M}_{2 n}$.

\section{Review and Open Problems}

In this section, we give an outline of the construction of $\hat{\mathcal{G}}^{(1)}-\mathrm{KdV}$ hierarchy (cf. [11,53]), explain the key steps needed in constructing curve flows whose differential invariants satisfy the $\hat{\mathcal{G}}^{(1)}-\mathrm{KdV}$, and give some open problems.

Let $G$ be a non-compact, real simple Lie group, $\mathcal{G}$ its Lie algebra, and

$$
\hat{\mathcal{G}}^{(1)}=\mathcal{L}(\mathcal{G})=\left\{\sum_{i \leq n_{0}} \xi_{i} \lambda^{i} \mid n_{0} \text { an integer, } \xi_{i} \in \mathcal{G}\right\}
$$

Let

$$
\hat{\mathcal{G}}_{+}^{(1)}=\left\{\sum_{i \geq 0} \xi_{i} \lambda^{i} \in \mathcal{L}(\mathcal{G})\right\}, \quad \hat{\mathcal{G}}_{-}^{(1)}=\left\{\sum_{i<0} \xi_{i} \lambda^{i} \in \mathcal{L}(\mathcal{G})\right\} .
$$

Then $\left(\hat{\mathcal{G}}_{+}^{(1)}, \hat{\mathcal{G}}_{-}^{(1)}\right)$ is a splitting of $\hat{\mathcal{G}}^{(1)}$.

Let $\left\{\alpha_{1}, \ldots, \alpha_{n}\right\}$ be a simple root system of $\mathcal{G}$, and $\mathcal{B}_{+}, \mathcal{B}_{-}, \mathcal{N}_{+}$the Borel subalgebras of $\mathcal{G}$ of non-negative roots, non-positive roots, and positive roots respectively. Let $B_{+}, B_{-}, N_{+}$ be connected subgroups of $G$ with Lie algebras $\mathcal{B}_{+}, \mathcal{B}_{-}, \mathcal{N}_{+}$respectively. Let

$$
J=\beta \lambda+b,
$$

where $b=-\sum_{i=1}^{n} \alpha_{i}$ and $\beta$ is the highest root. 
The construction of $\hat{C}_{n}^{(1)}$-hierarchy in Section 3 works for $\hat{\mathcal{G}}^{(1)}$ except that the generating function $P(q, \lambda)$ in Proposition 2 should satisfy

$$
\left\{\begin{array}{l}
{\left[\partial_{x}+b+q, S(q, \lambda)\right]=0,} \\
m(S(q, \lambda))=0
\end{array}\right.
$$

where $m$ is the minimal polynomial of $J$ defined by (70).

Assume that there is a sequence of increasing positive integers $\left\{n_{j} \mid j \geq 1\right\}$ such that $J^{n_{j}}$ lies in $\hat{\mathcal{G}}_{+}^{(1)}$ for all $j \geq 1$. Write

$$
S^{n_{j}}(q, \lambda)=\sum_{i} S_{n_{j}, i}(q) \lambda^{i}
$$

Then the $n_{j}$-th flow in the $\hat{\mathcal{G}}^{(1)}$-hierarchy is

$$
q_{t}=\left[\partial_{x}+b+q, S_{n_{j}, 0}(q)\right]
$$

for $q: \mathbb{R}^{2} \rightarrow \mathcal{B}_{+}$.

Using the same kind of proofs for the $\hat{C}_{n}^{(1)}$-hierarchy, we obtain the following properties of the $\hat{\mathcal{G}}^{(1)}$-hierarchy:

(i) The existence of a Lax pair, $\left[\partial_{x}+J+q, \partial_{t}+\left(S^{n_{j}}(q, \lambda)\right)_{+}\right]=0$ for $(72)$.

(ii) The $\hat{\mathcal{G}}^{(1)}$-flows are invariant under the gauge action of $C^{\infty}\left(\mathbb{R}, N_{+}\right)$on $C^{\infty}\left(\mathbb{R}, \mathcal{B}_{+}\right)$.

(iii) If we find a linear subspace $V$ of $\mathcal{G}$ such that $C^{\infty}(\mathbb{R}, V)$ is a cross-section of the gauge action of $C^{\infty}\left(\mathbb{R}, N_{+}\right)$on $C^{\infty}\left(\mathbb{R}, \mathcal{B}_{+}\right)$. Then we can push down the $\hat{\mathcal{G}}^{(1)}$-flows to the cross-section $C^{\infty}(\mathbb{R}, V)$ along gauge orbits and obtain a $\hat{\mathcal{G}}^{(1)}-\mathrm{KdV}$ hierarchy on $C^{\infty}(\mathbb{R}, V)$. Moreover, there exists a polynomial differentials $\xi_{j}(u)$ such that the $n_{j}$-th flow in the $\hat{\mathcal{G}}^{(1)}-\mathrm{KdV}$ hierarchy is

$$
u_{t}=\left[\partial_{x}+b+u, S_{n_{j}, 0}(u)-\xi_{j}(u)\right] .
$$

The $\hat{\mathcal{G}}^{(1)}$-KdV hierarchies constructed from two different cross- sections are not the same but are gauge equivalent.

(iv) The bi-Hamiltonian structure $\left(\{,\}_{1},\{,\}_{2}^{\wedge}\right)$ on $C^{\infty}\left(\mathbb{R}, \mathcal{B}_{+}\right)$is given by (66), (67).

(v) The Poisson structures $\{,\}_{1}^{\wedge}$ and $\{,\}_{2}$ are invariant under the gauge group action. So there is an induced bi-Hamiltonian structure on $C^{\infty}\left(S^{1}, V\right)$ for the $\hat{\mathcal{G}}^{(1)}-\mathrm{KdV}$ hierarchy, which will be denoted by $\left(\{,\}_{1},\{,\}_{2}\right)$.

(vi) $F_{n_{j}}(q)=-\oint\left(S_{n_{j},-1}(q) \beta\right) \mathrm{d} x$ is the Hamiltonian for the $n_{j}$-th flow with respect to $\{,\}_{2}$. Although properties (i)-(vi) can be proved in a unified way for any $\hat{\mathcal{G}}^{(1)}$, the following results need to be proved case by case depending on $\mathcal{G}$ :

(1) Find a linear subspace $V$ such that $C^{\infty}(\mathbb{R}, V)$ is a cross-section of the gauge action of $C^{\infty}\left(\mathbb{R}, N_{+}\right)$on $C^{\infty}\left(\mathbb{R}, \mathcal{B}_{+}\right)$.

(2) Suppose $\mathcal{G}$ is a subalgebra of $g l(n)$ and $C^{\infty}(\mathbb{R}, V)$ is a cross-section of the gauge action. We consider the following class of curves in $\mathbb{R}^{n}$ :

$$
\begin{gathered}
\mathcal{M}=\left\{g e_{1} \mid g \in C^{\infty}(\mathbb{R}, G) \text { satisfying } g^{-1} g_{x}=b+u,\right. \\
\text { for some } \left.u \in C^{\infty}(\mathbb{R}, V)\right\} .
\end{gathered}
$$

Find geometric properties of curves in $\mathcal{M}$ that characterize $\gamma \in \mathcal{M}$ (so $g$ is the moving frame and $u$ is the differential invariant of $\gamma$ under the group $G$ ). For example, for the $\hat{C}_{n}^{(1)}$ case, it is easy to see that if $\gamma \in \mathcal{M}$, then $\gamma$ is Lagrangian (see Definition 1). Conversely, if $\gamma$ is Lagrangian then $g \in \mathcal{M}$.

(3) Identify the tangent space of $\mathcal{M}$ at $\gamma$. 
(4) Show that

$$
\gamma_{t}=g S_{n_{j}, 0}(u) e_{1}
$$

is a flow on $\mathcal{M}$, i.e., the right hand side is tangent to $\mathcal{M}$.

(5) Show that if $\gamma(x, t)$ is a solution of (74), then the differential invariants $u(\cdot, t)$ satisfies the $\hat{\mathcal{G}}^{(1)}-\mathrm{KdV}$ flow (73). This also gives a natural interpretation of the $\hat{\mathcal{G}}^{(1)}-\mathrm{KdV}$.

(6) Write down the formula for the induced bi-Hamiltonian structure for the $\hat{\mathcal{G}}^{(1)}-\mathrm{KdV}$ hierarchy.

(7) We pull back the bi-Hamiltonian structure on $C^{\infty}\left(S^{1}, V\right)$ to $\mathcal{M}$ via the curvature map $\Psi: \mathcal{M} \rightarrow C^{\infty}\left(S^{1}, V\right)$ defined by $\Psi(\gamma)=u$ the differential invariant of $\gamma$. Then soliton properties of $\hat{\mathcal{G}}^{(1)}-\mathrm{KdV}$ can be also pulled back to the curve flows (74) on $\mathcal{M}$.

(8) Prove an analogue of Theorem 5, i.e., if $C: \mathbb{R} \rightarrow \mathcal{G}$ satisfies $\left[\partial_{x}+b+u, C\right] \in$ $C^{\infty}(\mathbb{R}, V)$, then

(a) $C$ is determined by $C e_{1}$,

(b) $C$ is determined by the projection of $C$ onto $V^{t}$, where $u \in C^{\infty}(\mathbb{R}, V)$.

We need this result to give a precise description of the tangent space of $\mathcal{M}$ at $\gamma$ and to write down the formula for the induced bi-Hamiltonian structure on $C^{\infty}(\mathbb{R}, V)$ for the $\hat{\mathcal{G}}^{(1)}-\mathrm{KdV}$ hierarchy.

(9) To construct Darboux transforms, we need to find rational maps $g: \mathbb{R} \rightarrow G_{\mathbb{C}}$ satisfies $\overline{g(\bar{\lambda})}=g(\lambda)$ with minimal number of poles and work out the factorization formula explicitly.

Let $\sigma$ be an involution of $\mathcal{G}$, and $\mathcal{K}, \mathcal{P}$ the $1,-1$ eigenspaces of $\sigma$. The $\hat{\mathcal{G}}^{(2)}$-hierarchy is constructed from the splitting $\left(\hat{\mathcal{G}}_{+}^{(2)}, \hat{\mathcal{G}}_{-}^{(2)}\right)$ of $\hat{\mathcal{G}}^{(2)}$, where

$$
\begin{aligned}
& \hat{\mathcal{G}}^{(2)}=\left\{\xi(\lambda)=\sum_{i} \xi_{i} \lambda^{i} \mid \overline{\xi(\bar{\lambda})}=\xi(\lambda), \sigma(\xi(-\lambda))=\xi(\lambda)\right\}, \\
& \hat{\mathcal{G}}_{+}^{(2)}=\hat{\mathcal{G}}^{(2)} \cap \hat{\mathcal{G}}_{+}^{(1)}, \quad \hat{\mathcal{G}}_{-}^{(2)}=\hat{\mathcal{G}}^{(2)} \cap \hat{\mathcal{G}}_{-}^{(1)} .
\end{aligned}
$$

Assume that there is a simple root system of $\mathcal{G}$ so that $\beta \in \mathcal{P}$ and $b \in \mathcal{K}$. Then $C^{\infty}\left(\mathbb{R}, \mathcal{K} \cap \mathcal{B}_{+}\right)$is invariant under the $\hat{\mathcal{G}}^{(1)}$-hierarchy. The $\hat{\mathcal{G}}^{(2)}$-hierarchy is the restriction of the $\hat{\mathcal{G}}^{(1)}$-hierarchy to $C^{\infty}\left(\mathbb{R}, \mathcal{K} \cap \mathcal{B}_{+}\right)$. Most properties of the $\hat{\mathcal{G}}^{(1)}$-hierarchy hold for the $\hat{\mathcal{G}}^{(2)}$-hierarchy except the bi- Hamiltonian structure $\{,\}_{1}^{\wedge}$ is zero on $C^{\infty}\left(S^{1}, \mathcal{K} \cap \mathcal{B}_{+}\right)$. To obtain the other Poisson structure, we need to review briefly a general method to construct a sequence of compatible Poisson structures from a bi-Hamiltonian structure: Let $\Xi_{i}$ denote the Poisson operator for $\{,\}_{i}^{\wedge}$ on $C^{\infty}\left(\mathbb{R}, \mathcal{B}_{+}\right)$, i.e., $\left(\Xi_{i}\right)_{q}: C^{\infty}\left(S^{1}, \mathcal{B}_{-}\right) \rightarrow C^{\infty}\left(S^{1}, \mathcal{B}_{+}\right)$is defined by

$$
\left\{F_{1}, F_{2}\right\}_{i}^{\wedge}(q)=\left\langle\left(\Xi_{1}\right)_{q}\left(\nabla F_{1}(q)\right), \nabla F_{2}(q)\right\rangle
$$

for $i=1,2$. It is known (cf. $[55,56])$ that

$$
\left\{F_{1}, F_{2}\right\}_{j}^{\wedge}(q)=\left\langle\left(\Xi_{j}\right)_{q}\left(\nabla F_{1}(q)\right), \nabla F_{2}(q)\right\rangle
$$

is again a Poisson structure and are compatible, where

$$
\Xi_{j}:=\Xi_{2}\left(\Xi_{1}^{-1} \Xi_{2}\right)^{j-2} .
$$

It can be checked that $\Xi_{2 i+1}=0$ on $C^{\infty}\left(S^{1}, \mathcal{K} \cap \mathcal{B}_{+}\right)$, and $\Xi_{2 i}$ is a Poisson structure for the $\hat{\mathcal{G}}^{(2)}$-hierarchy for all $i \geq 1$. So $\left(\{,\}_{2},\{,\}_{4}^{\wedge}\right)$ is a bi-Hamiltonian structure for the $\hat{\mathcal{G}}^{(2)}$-hierarchy and it induces a bi-Hamiltonian structure $\left(\{,\}_{2},\{,\}_{4}\right)$ for the $\hat{\mathcal{G}}^{(2)}$ KdV hierarchy.

Finally we give a list of open problems:

$\diamond \quad$ Find integrable curve flows on $\mathbb{R}^{2 n, 1}$ whose differential invariants satisfy the $\hat{B}_{n}^{(1)}-\mathrm{KdV}$ flows.

$\diamond \quad$ Find integrable curve flows on $\mathbb{R}^{k, 2 n-k}$ whose differential invariants satisfy the $\hat{D}_{n}^{(1)}-\mathrm{KdV}$ flows. 
$\diamond \quad$ Find integrable curve flows on $\mathbb{R}^{2 n}$ whose differential invariants satisfy the $\hat{D}_{n}^{(2)}-\mathrm{KdV}$ flows.

$\diamond \quad$ Find integrable curve flows on $\mathbb{R}^{8}$ whose differential invariants satisfy the $\hat{D}_{4}^{(3)}-\mathrm{KdV}$ flows.

$\diamond \quad$ Find integrable curve flows on $\mathbb{R}^{7}$ whose differential invariants satisfy the $\hat{G}_{2}^{(1)}-\mathrm{KdV}$ flows.

$\diamond \quad$ Calini and Ivey constructed finite gap solutions for the VFE in [57]. It would be interesting to construct finite-gap solutions for central affine curve flows, isotropic curve flows, and Lagrangian curve flows.

$\diamond \quad$ The Gauss-Codazzi equations of submanifolds occurring in soliton theory are often given by the first level flows of the soliton hierarchy, i.e., the commuting flows generated by degree one (in $\lambda$ ) elements in the vacuum sequence. It would be interesting to see whether the flows of the $\hat{\mathcal{G}}^{(1)}$-KdV hierarchy generated by degree one elements in the vacuum sequence also arise as the Gauss-Codazzi equations for some class of submanifolds.

Author Contributions: Both authors are equally responsible for all results in this paper. All authors have read and agreed to the published version of the manuscript.

Funding: This research received no external funding.

Conflicts of Interest: The authors declare no conflict of interest.

\section{References}

1. Zabusky, N.J.; Kruskal, M.D. Interaction of solitons in a collisionless plasma and the recurrence of initial states. Phys. Rev. Lett. 1965, 15, 240-243. [CrossRef]

2. Gardner, C.S.; Greene, J.M.; Kruskal, M.D.; Miura, R.M. Method for solving the Korteweg-de Vries equation. Phys. Rev. Lett. 1967, 19, 1095-1097. [CrossRef]

3. Lax, P.D. Integrals of nonlinear equations of evolution and solitary waves. Commun. Pure Appl. Math. 1968, 21, 467-490. [CrossRef]

4. Zakharov, V.E.; Faddeev, L.D. Korteweg-de Vries equation, a completely integrable Hamiltonian system. Func. Anal. Appl. 1971, 5, 280-287. [CrossRef]

5. Zakharov, V.E.; Shabat, A.B. Exact theory of two-dimensional self-focusing and one-dimensional of waves in nonlinear media. Sov. Phys. JETP 1972, 34, 62-69.

6. Adler, M. On a trace functional for formal pseudo-differential operators and the symplectic structure of the Korteweg-de Vries Type Equations. Invent. Math. 1979, 50, 219-248. [CrossRef]

7. Adler, M.; van Moerbeke, P. Completely integrable systems, Euclidean Lie algebras and curves. Adv. Math. 1980, 38, 267-317. [CrossRef]

8. Kostant, B. The solution to a generalized Toda lattice and representation theory. Adv. Math. 1979, 34, 195-338. [CrossRef]

9. Symes, W.W. Systems of Toda type, Inverse spectral problems, and representation theory. Invent. Math. 1980, 59, 13-51. [CrossRef]

10. Kupershmidt, B.A.; Wilson, G. Modifying Lax equations and the second Hamiltonian structure. Invent. Math. 1981, 62, 403-436. [CrossRef]

11. Drinfeld, V.G.; Sokolov, V.V. Lie algebras and equations of Korteweg-de Vries type. (Russ.) Curr. Probl. Math. 1984, $24,81-180$.

12. Ablowitz, M.J.; Clarkson, P.A. Solitons, Nonlinear Evolution Equations and Inverse Scattering; London Mathematical Society Lecture Note Series, 149; Cambridge University Press: Cambridge, UK, 1991.

13. Beals, R.; Deift, P.; Tomei, C. Direct and Inverse Scattering on the Line, Mathematical Surveys and Monographs; American Mathematical Society: Providence, RI, USA, 1988; Volume 28.

14. Dickey, L.A. Soliton Equations and Hamiltonian Systems, 2nd ed.; Advanced Series in Mathematical Physics; World Scientific Publishing Co. Inc.: River Edge, NJ, USA, 2003; p. 26.

15. Faddeev, L.D.; Takhtajan, L.A. Hamiltonian Methods in the Theory of Solitons; Springer: Berlin, Germany, 1987.

16. Magri, F. Eight Lectures on Integrable Systems; Written in Collaboration with P. Casati, G. Falqui and M. Pedroni, Lecture Notes in Phys 495, Integrability of Nonlinear Systems; Springer: Berlin, Germany, 1997; pp. 256-296.

17. Palais, R.S. The symmetries of solitons. Bull. AMS 1997, 34, 339-403. [CrossRef]

18. Terng, C.L.; Uhlenbeck, K. Poisson actions and scattering theory for integrable systems. Surv. Differ. Geom. 1999, 4, 315-402. [CrossRef]

19. Darboux, G. Leçons sur les Systèmes Orthogonaux et les Coordonnées Curvilignes. Principes de Géométrie Analytique, 2nd ed.; GauthierVillars: Paris, France, 1910.

20. Tenenblat, K.; Terng, C.L. Bäcklund's theorem for n-dimensional submanifolds of $R^{2 n-1}$. Ann. Math. 1980, 111, 477-490. [CrossRef] 
21. Terng, C.L. A higher dimensional generalization of the sine-Gordon equation and its soliton theory. Ann. Math. 1980, 111, 491-510. [CrossRef]

22. Ferus, D.; Pedit, F. Isometric immersions of space forms and soliton theory. Math. Ann. 1996, 305, 329-342. [CrossRef]

23. Tenenblat, K. Bäcklund's theorem for submanifolds of space forms and a generalized wave equation. Bull. Soc. Brasil. Math. 1985, 16, 67-92. [CrossRef]

24. Terng, C.L.; Wang, E. Transformations of flat Lagrangian immersions and Egoroff nets. Asian J. Math. 2008, 12, 99-119. [CrossRef]

25. Donaldson, N.; Terng, C.L. Conformally flat submanifolds in spheres and integrable systems. Tohoku Math. J. 2011, 63, 277-302. [CrossRef]

26. Burstall, F.E. Isothermic Surfaces: Conformal Geometry, Clifford Algebras and Integrable Systems; Integrable Systems, Geometry, and Topology, AMS/IP Stud. Adv. Math.; American Mathematical Society: Providence, RI, USA, 2006; Volume 36, pp. 1-82.

27. Bruck, M.; Du, X.; Park, J.; Terng, C.L. Submanifold geometry of real Grassmannian systems. Mem. AMS 2002, $155,102$.

28. Donaldson, N.; Terng, C.L. Isothermic submanifolds. J. Geom. Anal. 2012, 22, 827-844. [CrossRef]

29. Terng, C.L. Geometries and Symmetries of Soliton Equations and Integrable Elliptic Systems; Surveys on Geometry and Integrable Systems; Advanced Studies in Pure Mathematics Mathematical Society of Japan: Tokyo, Japan, 2008; Volume 51, pp. 401-488.

30. Hasimoto, R. A soliton on a vortex filament. J. Fluid Mech. 1972, 51, 477-485. [CrossRef]

31. Terng, C.L. Dispersive Geometric Curve Flows; Surveys in Differential Geometry 2014. Regularity and Evolution of Nonlinear Equations, Surv. Differ. Geom.; International Press: Somerville, MA, USA, 2015; Volume 19, pp. 179-229.

32. Langer, J.; Perline, R. Poisson geometry of the filament equation. J. Nonlinear Sci. 1991, 1, 71-93. [CrossRef]

33. Langer, J.; Perline, R. Local geometric invariants of integrable evolution equations. J. Math. Phys. 1994, 35, 1732-1737. [CrossRef]

34. Langer, J.; Perline, P. Geometric realizations of Fordy-Kulish non- linear Schrd̈inger systems. Pac. J. Math. 2000, 195, 157-178. [CrossRef]

35. Doliwa, A.; Santini, P.M. An elementary geometric characterization of the integrable motions of a curve. Phys. Lett. A 1994, 185, 373-384. [CrossRef]

36. Ferapontov, E.V. Isoparametric hypersurfaces in spheres, integrable non-diagonalizable systems of hydrodynamic type, and Nwave systems. Differ. Geom. Appl. 1995, 5, 335-369. [CrossRef]

37. Yasui, Y.; Sasaki, N. Differential geometry of the vortex filament equation. J. Geom. Phys. 1998, 28, 195-207. [CrossRef]

38. Chou, K.-S.; Qu, C. Integrable equations arising from motions of plane curves. Phys. D 2002, 162, 9-33. [CrossRef]

39. Chou, K.-S.; Qu, C. Integrable equations arising from motions of plane curves, II. J. Nonlinear Sci. 2003, 13, 487-517. [CrossRef]

40. Anco, S.C. Hamiltonian flows of curves in $G / S O(N)$ and vector soliton equations of mKdV and sine-Gordon type. SIGMA Symmetry Integr. Geom. Methods Appl. 2006, 2, 044. [CrossRef]

41. Sanders, J.A.; Wang, J.P. Integrable systems in n-dimensional Riemannian geometry. Mosc. Math. J. 2003, 3, 1369-1393. [CrossRef]

42. Terng, C.L.; Thorbergsson, G. Completely integrable curve flows on adjoint orbits. Results Math. 2001, 40, 286-309. [CrossRef]

43. Terng, C.-L.; Uhlenbeck, C. Schrödinger flows on Grassmannians, Integrable systems, geometry, and topology. AMS/IP Stud. Adv. Math. 2006, 36, 235-256.

44. Terng, C.L. Geometric Airy curve flow on $\mathbb{R}^{n}$. Surv. Differ. Geom. 2018, 23, 277-303. [CrossRef]

45. Mari Beffa, G. Poisson geometry of differential invariants of curves in some non- semi-simple homogeneous spaces. Proc. Am. Math. Soc. 2006, 134, 779-791. [CrossRef]

46. Mari Beffa, G. Geometric Hamiltonian structures on flat semisimple homogeneous manifolds. Asian J. Math. 2008, 12, 1-33. [CrossRef]

47. Pinkall, U. Hamiltonian flows on the space of star-shaped curves. Results Math. 1995, 27, 328-332. [CrossRef]

48. Calini, A.; Ivey, T.; Marí Beffa, G. Integrable flows for starlike curves in centroaffine space. SIGMA Symmetry Integr. Geom. Methods Appl. 2013, 9, 21. [CrossRef]

49. Terng, C.L.; Wu, Z. N-dimension central affine curve flows. J. Differ. Geom. 2019, 111, 145-189. [CrossRef]

50. Terng, C.L.; $\mathrm{Wu}, \mathrm{Z}$. Isotropic geometric curve flows on $\mathbb{R}^{n+1, n}$. to appear in Comm. Anal. Geom.

51. Calini, A.; Ivey, T.; Mari Beffa, G. Remarks on KdV-type flows on star-shaped curves. Physica D 2009, 238, 788-797. [CrossRef]

52. Terng, C.L.; Wu, Z. Central affine curve flow on the plane. J. Fixed Point Theory Appl. 2013, 14, 375-396. [CrossRef]

53. Terng, C.L.; Uhlenbeck, K. The $n \times n$ KdV hierarchy. J. Fixed Point Theory Appl. 2011, 10, 37-61. [CrossRef]

54. Terng, C.L.; Uhlenbeck, K. Bäcklund transformations and loop group actions. Comm. Pure Appl. Math. 2000, 53, 1-75. [CrossRef]

55. Magri, F. On the geometry of Soliton equations. Acta Aplic. Math. 1995, 41, 247-270.

56. Terng, C.L. Soliton equations and differential geometry. J. Differ. Geom. 1997, 45, 407-445. [CrossRef]

57. Calini, A.; Ivey, Finite-Gap Solutions of the Vortex Filament Equation: Isoperiodic Deformations. J. Nonlinear Sci. 2007, 17, 527-567. [CrossRef] 